\title{
INEEL Advanced Radiotherapy Research Program Annual Report 2002
}

\author{
J. R. Venhuizen
}

May 2003

Idaho National Engineering and Environmental Laboratory Bechtel BWXT Idaho, LLC 


\title{
INEEL Advanced Radiotherapy Research Program Annual Report 2002
}

\author{
J. R. Venhuizen
}

May 2003

Idaho National Engineering and Environmental Laboratory Idaho Falls, Idaho 83415

\author{
Prepared for the \\ U.S. Department of Energy \\ Office of Science \\ Under DOE Idaho Operations Office \\ Contract DE-AC07-99ID13727
}




\begin{abstract}
This report summarizes the activities and major accomplishments for the Idaho National Engineering and Environmental Laboratory (INEEL) Advanced Radiotherapy Research Program for calendar year 2002. Topics covered include computational dosimetry and treatment planning software development, medical neutron source development and characterization, and boron analytical chemistry.
\end{abstract}




\section{CONTENTS}

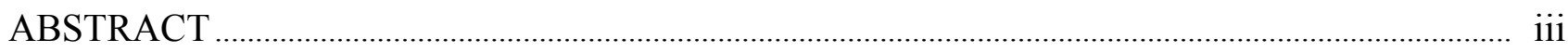

ACRONYMS AND ABBREVIATIONS ..................................................................... ix

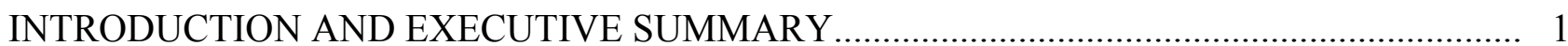

BASELINE ANALYTICAL CHEMISTRY AND DOSIMETRY SOFTWARE SUPPORT

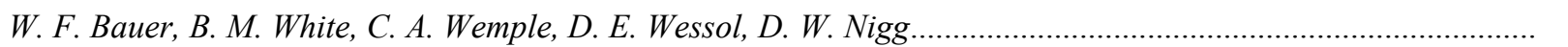

3

RADIATION TREATMENT PLANNING SOFTWARE DEVELOPMENT

C. A. Wemple, D.E. Wessol, J. J. Cogliati, C. Frederickson, M. L. Milvich, G. J. Harkin,

M. A. Descalle, J. Lehmann, T. Flickinger, C. L. Hartmann Siantar

NEUTRONIC PERFORMANCE ASSESSMENT OF AN EPITHERMAL NEUTRON

BEAM FOR PRECLINICAL BNCT RESEARCH AT WASHINGTON STATE UNIVERSITY

D. W. Nigg, C. A. Wemple, J. R. Venhuizen, J. K. Hartwell, G. E. Tripard, S. Sharp, K. Fox, P. R. Gavin

TRANSPORT BENCHMARKS FOR PHYSICAL DOSIMETRY TO SUPPORT DEVELOPMENT OF FAST-NEUTRON THERAPY WITH NEUTRON CAPTURE AUGMENTATION

D. W. Nigg, J. K. Hartwell, C. A. Wemple, R. Risler 38

COLLABORATIVE IN-PHANTOM PERFORMANCE CHARACTERIZATION OF THE CNEA HYPERTHERMAL NEUTRON BEAM FACILITY FOR BNCT

D. W. Nigg, H. Blaumann, O. C. Larrieu, J. Longhino, C. A. Wemple, S. Ribeir, M. Arribere. 42 


\section{FIGURES}

\section{RADIATION TREATMENT PLANNING SOFTWARE DEVELOPMENT}

Figure 1. MINERVA data flow design

Figure 2. Comparison between absorbed fractions for MINERVA prototype, EGS4 and MCNP for $100 \mathrm{keV}, 400 \mathrm{keV}, 1 \mathrm{MeV}$, and $4 \mathrm{MeV}$ electron spherical sources

Figure 3. Comparison between MINERVA prototype and EGS4 prediction of absorbed fractions for several spherical sources with radioisotopes of interest.

Figure 4. Dose distribution of ${ }^{131} \mathrm{I}$ in a kidney obtained with 3D Monte Carlo simulations 12

Figure 5. Dose distribution of ${ }^{131} \mathrm{I},{ }^{67} \mathrm{Cu}$, and ${ }^{90} \mathrm{Y}$ in a kidney obtained with 3D Monte Carlo simulations

\section{NEUTRONIC PERFORMANCE ASSESSMENT OF AN EPITHERMAL NEUTRON BEAM FOR PRECLINICAL BNCT RESEARCH AT WASHINGTON STATE UNIVERSITY}

Figure 1. Elevation plan of the WSU TRIGA reactor facility ................................................... 17

Figure 2. WSU column assembly, with epithermal neutron filter in place.................................... 18

Figure 3. Approximate WSU beam stop and treatment room arrangement .................................. 18

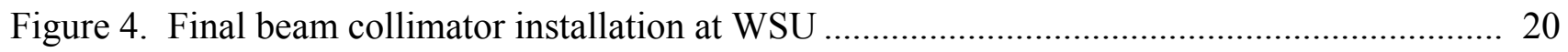

Figure 5. Lithiated polyethylene neutron suppression shield placement ........................................ 20

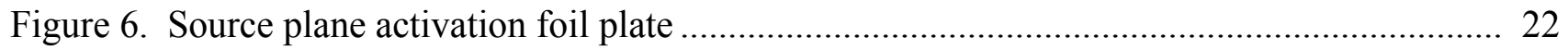

Figure 7. Boron sphere used to suppress low-energy neutron flux................................................. 23

Figure 8. RemSpec ${ }^{\mathrm{TM}}$ superheated nucleation spectrometer unit positioned in the source

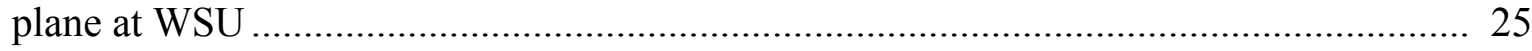

Figure 9. Beam aperture plates and various Lucite phantoms used at WSU .................................. 26

Figure 10. Lithiated polyethylene beam aperture plate arrangement for the $10.16 \mathrm{~cm}$ diameter beam exit aperture at WSU. 


\section{FIGURES (Continued)}

\section{NEUTRONIC PERFORMANCE ASSESSMENT OF AN EPITHERMAL NEUTRON BEAM FOR PRECLINICAL BNCT RESEARCH AT WASHINGTON STATE UNIVERSITY (CONTINUED)}

Figure 11. Foil positioning device for exit aperture spectral measurements at WSU 27

Figure 12. Foil wheel in the $10.16 \mathrm{~cm}$ beam exit aperture at WSU 27

Figure 13. Boron sphere positioned in the $10.16 \mathrm{~cm}$ beam exit aperture at WSU..... 28

Figure 14. INEEL Lucite phantom 29

Figure 15. Positioning of the Lucite phantom in the WSU beam exit aperture 29

Figure 16. Unfolded free beam neutron spectrum in the source plane obtained by direct fitting for the WSU epithermal neutron beam facility

Figure 17. Unfolded free beam neutron spectrum in the $10.16 \mathrm{~cm}$ diameter beam aperture obtained by direct fitting for the WSU epithermal neutron beam facility .... 33

Figure 18. Results of the RemSpec ${ }^{\mathrm{TM}}$ measurements for the WSU epithermal neutron beam ...... 33

Figure 19. Fluxes in the INEEL cylindrical Lucite phantom irradiated in the $10.16 \mathrm{~cm}$ aperture of the WSU epithermal neutron beam facility .....

Figure 20. Water phantom at WSU, with detail of paired ion chamber arrangement

\section{TRANSPORT BENCHMARKS FOR PHYSICAL DOSIMETRY TO SUPPORT DEVELOPMENT OF FAST-NEUTRON THERAPY WITH NEUTRON CAPTURE AUGMENTATION}

Figure 1. University of Washington neutron therapy treatment facility with the large cylindrical Lucite phantom in position

Figure 2. Saturation activities for Mn-56 in the small and large phantoms 40

Figure 3. Saturation activities for several gold isotopes in the small phantom 40 
FIGURES (Continued)

\section{COLLABORATIVE IN-PHANTOM PERFORMANCE CHARACTERIZATION OF THE CNEA HYPERTHERMAL NEUTRON BEAM FACILITY FOR BNCT}

Figure 1. RA-6 neutron beam filtering and extraction component design .................................... 42

Figure 2. RA-6 epithermal neutron beam port....................................................................... 43

Figure 3. Water phantom positioned in the RA-6 beam port....................................................... 44

Figure 4. Measured thermal and above-thermal neutron fluxes along the central axis of the CNEA water phantom ............................................................................... 45

Figure 5. Measured thermal and above-thermal neutron fluxes along the radial dimension at an axial depth of $6 \mathrm{~mm}$ in the CNEA water phantom

\section{TABLES}

\section{RADIATION TREATMENT PLANNING SOFTWARE DEVELOPMENT}

Table 1. Comparison of specific absorbed fractions calculated with the MINERVA prototype code and the specific absorbed fractions given in revised MIRD pamphlet $5 \mathrm{f}$ for $100 \mathrm{keV}$ photons emitted by source organs of a heterogeneous phantom

\section{NEUTRONIC PERFORMANCE ASSESSMENT OF AN EPITHERMAL NEUTRON BEAM} FOR PRECLINICAL BNCT RESEARCH AT WASHINGTON STATE UNIVERSITY

Table 1. Activation interactions and foils used for the INEEL/WSU epithermal-neutron beam measurements.

Table 2. Foil saturation activities measured at a WSU reactor power of $1 \mathrm{MW}$ 30 


\section{ACRONYMS AND ABBREVIATIONS}

ANL Argonne National Laboratory

API Application Programming Interface

BLOB Binary Large Object

BNCT Boron Neutron Capture Therapy

BNL Brookhaven National Laboratory

$\mathrm{Bq} \quad$ Becquerel

cGy centiGray

cm centimeter

CNEA National Atomic Energy

Commission of Argentina

CPU central processor unit

CRADA Cooperative Research and

Development Agreement

CSDA continuous slowing down approximation

CT Computed Tomography

CY calendar year

DOE Department of Energy

DORT Discrete Ordinates Radiation Transport

E energy

eTCP effective tumor control probability

$\mathrm{eV} \quad$ electron volt
FiR 1 Finnish Research Reactor

FNT fast neutron therapy

g gram

Gy $\quad$ Gray

HPGe high-purity germanium

ICP-AES inductively coupled plasmaatomic emission spectrometry

in. inch

INEEL Idaho National Engineering and Environmental Laboratory

$\mathrm{I} / \mathrm{O} \quad$ input/output

JAR Java archive file

JDBC Java database connectivity

JDOM Java document object module

keV kilo-electron volt

kW kilowatt

LLNL Lawrence Livermore National Laboratory

m meter

MCNP Monte Carlo N-Particle

$\mathrm{MeV} \quad$ Megaelectron volt

mg milligram

MINERVA Modality-Inclusive Environment for Radiotherapeutic Variable Analysis 


\section{ACRONYMS AND ABBREVIATIONS (Continued)}

\begin{tabular}{|c|c|c|c|}
\hline MIRD & $\begin{array}{l}\text { Medical Internal Radiation } \\
\text { Dose }\end{array}$ & Rtpe & $\begin{array}{l}\text { Radiation treatment planning } \\
\text { environment }\end{array}$ \\
\hline $\mathrm{mm}$ & millimeter & $\mathrm{s}$ & second \\
\hline MML & MINERVA markup language & $\mathrm{SC}$ & Office of Science \\
\hline $\operatorname{mrad}$ & millirad & SDD & Superheated Drop Detector \\
\hline MSU & Montana State University & SERA & $\begin{array}{l}\text { Simulation Environment for } \\
\text { Radiotherapy Applications }\end{array}$ \\
\hline $\begin{array}{l}\text { MW } \\
\mathrm{n}\end{array}$ & $\begin{array}{l}\text { Megawatt } \\
\text { neutron }\end{array}$ & SWT & $\begin{array}{l}\text { Southwest Texas State } \\
\text { University }\end{array}$ \\
\hline $\mathrm{NCT}$ & neutron capture therapy & TCP & tumor control probability \\
\hline NIST & $\begin{array}{l}\text { National Institute of Standards } \\
\text { and Technology }\end{array}$ & $\mathrm{TI}$ & therapeutic index \\
\hline NNSA & $\begin{array}{l}\text { National Nuclear Security } \\
\text { Administration }\end{array}$ & TRIGA & $\begin{array}{l}\text { Transient Reactor Irradiator- } \\
\text { General Atomics }\end{array}$ \\
\hline NTCP & $\begin{array}{l}\text { normal tissue complication } \\
\text { probability }\end{array}$ & UCLA & $\begin{array}{l}\text { University of California, Los } \\
\text { Angeles }\end{array}$ \\
\hline NTL & $\begin{array}{l}\text { Neutron Therapies, Limited } \\
\text { Liability Company }\end{array}$ & UCSF & $\begin{array}{l}\text { University of California, San } \\
\text { Francisco }\end{array}$ \\
\hline & & UI & user interface \\
\hline QA & Quality assurance & UT & University of Tennessee \\
\hline QSH & $\begin{array}{l}\text { standard electronic image } \\
\text { format }\end{array}$ & UW & University of Washington \\
\hline RBE & $\begin{array}{l}\text { relative biological } \\
\text { effectiveness }\end{array}$ & WSU & Washington State University \\
\hline RIT & radioimmunotherapy & XML & extensible markup language \\
\hline ROI & region of interest & $3 \mathrm{D}$ & three-dimensional \\
\hline & & $\sigma$ & standard deviation \\
\hline
\end{tabular}




\section{INEEL ADVANCED RADIOTHERAPY RESEARCH PROGRAM ANNUAL REPORT 2002}

\section{INTRODUCTION AND EXECUTIVE SUMMARY}

The Idaho National Engineering and Environmental Laboratory (INEEL) Advanced Radiotherapy Research Program completed its $16^{\text {th }}$ year under the sponsorship of the U.S. Department of Energy (DOE), Office of Science (SC). The program underwent a major external peer review during calendar year (CY) 2001. As a result of this review, many ongoing activities were continued, a few others were reprioritized, and a new activity focused on collaboration with Lawrence Livermore National Laboratory (LLNL), in the field of advanced treatment planning software development, was initiated during the fourth quarter of that year. This report includes detailed articles describing major INEEL activities and accomplishments during CY 2002.

A primary program goal has been, and continues to be, participation in the establishment of neutron capture therapy (NCT) as a clinically useful innovative radiotherapy in and of itself, or as an adjunct to fast-neutron therapy (FNT). In addition, a new task involving the development of advanced multi-modality computational dosimetry and treatment planning software with an initial emphasis on molecular targeted radionuclide applications was introduced during CY 2001, and significant progress was made during CY 2002. Various program goals were accomplished through applications of supportive research and development and technology deployment in the fields of chemistry, radiation physics and dosimetry, and neutron source design and demonstration. Many activities have been performed in close collaboration with other DOE national laboratories and major universities. Where a benefit to the U.S. NCT effort is realized, the INEEL also maintains formal cooperative arrangements with certain foreign collaborators, specifically in The Netherlands, Finland, Sweden, and Argentina. Finally, in light of the stated goals of DOE for technology transfer and commercialization, some aspects of the Program were operated in partnership with private U.S. industry through a Cooperative Research and Development Agreement (CRADA), as well as through several technology licensing agreements. These arrangements have resulted in substantial leveraging of DOE-SC funding, especially in the important area of boron agent development.

The historical INEEL role as a center for quantitative boron analysis and radiation treatment planning software development and deployment continued during CY 2002. Biological samples and boron agent samples submitted to the INEEL by federally supported NCT research organizations were analyzed for boron content in accordance with well-established standards, and the results were reported back to the submitting organizations. The internationally recognized INEEL SERA (Simulation Environment for Radiotherapy Applications) computational dosimetry and treatment planning software system continued to be supported during the year, and several new national and international licensees joined the SERA user community. The initial release of SERA occurred in 1998, considerable refinement and additional feature development took place in 1999 and 2000, and the distribution version was stabilized in 2001. NCT and FNT research centers worldwide continue to serve as benchmarking and testing institutions for SERA, providing extremely valuable feedback and suggestions for improvement. 
With the completion of SERA, the developmental focus is now on the new MINERVA (Modality Inclusive Environment for Radiotherapeutic Variable Analysis) multimodality radiotherapy computational dosimetry treatment planning system, in collaboration with LLNL. This effort will take advantage of the many benefits of using the Java programming language to create a "next-generation" system for a wide variety of research applications. Initial coding for three of the five modality-independent modules (overall system control, medical image manipulation, and patient geometry reconstruction) was completed, and work was started on establishing the detailed requirements for the other two (computational sequence control and dose reporting). A prototype source definition module for targeted radionuclides was also prepared and work also started at LLNL on coupling this module to the corresponding radiation transport computation module.

INEEL also continued its efforts toward the development of neutron sources useful for NCT and NCT-augmented FNT. This has been done under collaborations with the University of Washington (UW) fast-neutron radiotherapy program, and with the Washington State University (WSU) Nuclear Radiation Center and School of Veterinary Medicine. Detailed experimental neutronic characterization of the two fast-neutron beams at the UW neutron facility began, with a focus on providing high-quality benchmark data for validation of advanced treatment planning for fast-neutron therapy with neutron capture augmentation. In addition, construction of a new epithermal neutron beam facility for preclinical research at WSU was completed during the year, and several beam characterization measurements were performed in a collaborative effort of INEEL and WSU.

Finally, in addition to the domestic NCT activities supported at the INEEL by DOE-SC, INEEL researchers continued a collaboration with the National Atomic Energy Commission of Argentina in the areas of NCT beam dosimetry and small-animal radiobiology. This collaboration is partially supported by the DOE National Nuclear Energy Security Agency (NNSA) with in-kind contributions for an INEEL CRADA partner, Neutron Therapies, LLC. 


\title{
BASELINE ANALYTICAL CHEMISTRY AND DOSIMETRY SOFTWARE SUPPORT
}

\author{
W.F. Bauer, B.M. White, C.A. Wemple, D.E. Wessol, and D.W. Nigg \\ Idaho National Engineering and Environmental Laboratory, Idaho Falls, ID
}

\section{INTRODUCTION}

Baseline analytical chemistry and software support functions continued during calendar year (CY) 2002. These activities included the determination of boron in a variety of biologically related samples submitted by various institutions, as well as maintenance and distribution of the Idaho National Engineering and Environmental Laboratory (INEEL) SERA (Simulation Environment for Radiotherapy Applications) computational dosimetry and treatment planning software for a wide range of national and international collaborators.

\section{DETERMINATION OF BORON IN BIOLOGICAL SAMPLES}

During CY 2002, 533 new samples were received for analysis by inductively coupled plasma atomic emission spectroscopy (ICP-AES) using standardized procedures established by INEEL scientists. With samples carried over from CY 2001 and additional Quality Assurance (QA) samples, the total number of samples analyzed came to 579. Of the samples received, 285 were from dogs and/or rodents from Boron Neutron Capture Therapy and related programs in Dr. P. Gavin's group at Washington State University (WSU), eight were of stock solutions to be sent to WSU and assorted other preparations from Dr. F. Hawthorne's group at University of California, Los Angeles (UCLA), one was from Neutron Therapies, LLC (NTL), 234 were from the University of California, San Francisco (UCSF), two from Southwest Texas State University (SWT), and three were from the University of Tennessee (UT). As the neutron beam at WSU comes on line and radiobiology studies resume, the anticipated number of samples from WSU is expected to increase dramatically. During CY 2003, samples are expected from UCLA, WSU, SWT, and from UT. Many of the samples from WSU are actually in support of the researchers from the other institutions who enlist the aid of the WSU group for boron biodistribution studies.

\section{SERA SOFTWARE MAINTENANCE}

The internationally-recognized INEEL/Montana State University (MSU) SERA ${ }^{1}$ computational dosimetry and treatment planning software for neutron radiotherapy applications continued to serve as a research tool for a variety of applications worldwide. SERA is used as the computational treatment-planning tool for the clinical neutron capture therapy (NCT) trials that are currently ongoing in Finland and Sweden. It is also being introduced into clinical use in connection with the NCT clinical project centered at the Commission of the European Communities, Joint Research Centre, Petten, The Netherlands (replacing an earlier INEEL/MSU product, BNCT_Rtpe). Additionally, SERA is currently under evaluation as a treatment planning system for use in the clinical fast-neutron therapy program at the University of Washington. Many other institutions use SERA for non-clinical applications as well. Active SERA licensees supported by INEEL under formal research agreements currently include: 
Lawrence Berkeley Laboratory

University of Washington

Massachusetts Institute of Technology

University of Tennessee

Purdue University

Medical Radiological Research Center, Russian Academy of Medical Science

Commission of the European Communities, Joint Research Centre, Petten (The Netherlands)

Technical Research Centre of Finland

University Hospital Birmingham NHS (England)

University of Essen (Germany)

Comision Nacional de Energia Atomica (Argentina)

Studsvik Nuclear AB (Sweden)

Kyoto University (Japan)

National Tsing Hua University (Taiwan)

Nuclear Research and Consultancy Group NRG (The Netherlands)

Medical University of Hannover (Germany)

STRELA State Unitary Enterprise (Russia)

Leipunsky State Scientific Center Institute (Russia)

There were a number of refereed publications involving SERA applications during the year. An invited article on computational dosimetry ${ }^{2}$ in general, and SERA in particular, was prepared and accepted for publication in the Journal of Neuro-Oncology early in 2003. A major independent validation study was completed by the Massachusetts Institute of Technology during the year, the results of which will be submitted for publication in the journal Medical Physics. Finally, the use of SERA was acknowledged by the research community in a number of articles appearing in the Proceedings of the Tenth International Symposium on Neutron Capture Therapy ${ }^{3}$, a major international meeting held during the year.

\section{REFERENCES}

1. D.E. Wessol, et al., "Simulation Environment for Radiotherapy Applications User's Manual," Idaho National Engineering and Environmental Laboratory, INEEL/EXT-02-00698, June 2002.

2. D.W. Nigg, "Computational Dosimetry and Treatment Planning Considerations for Neutron Capture Therapy," J. Neuro-Oncology, 62: 75-86, 2003.

3. W. Sauerwein, R. Moss, A. Wittig (eds.), "Research and Development in Neutron Capture Therapy," Monduzzi Editore S.p.A., Bologna, Italy, 2002. 


\title{
RADIATION TREATMENT PLANNING SOFTWARE DEVELOPMENT
}

\author{
C.A. Wemple ${ }^{1}$, D.E. Wessol ${ }^{1}$, J.J. Cogliati ${ }^{2}$, C. Frederickson ${ }^{2}$, M. L. Milvich ${ }^{2}$, G.J. Harkin ${ }^{2}$, \\ M.A. Descalle ${ }^{3}$, J. Lehmann ${ }^{3}$, T. Flickinger ${ }^{3}$, and C.L. Hartmann Siantar ${ }^{3}$ \\ 1. Idaho National Engineering and Environmental Laboratory, Idaho Falls, ID \\ 2. Montana State University, Bozeman, MT \\ 3. Lawrence Livermore National Laboratory, Livermore, CA
}

\section{INTRODUCTION}

The MINERVA ${ }^{1}$ (Modality-Inclusive Environment for Radiotherapeutic Variable Analysis) project entered its second year of design and development with a focus shift towards code implementation for modules where design issues have been largely resolved. Certain remaining unresolved design issues mainly concern the planning and reporting modules, including control of the source and transport plugins and dose display methods. Final resolution of many of these issues will involve additional definition of functionality based on clinical feedback. Thus, the decision was made to focus coding development on the Patient, Image, and Model modules and, during calendar year (CY) 2003, to develop rapid prototypes for the other modules. These modules and prototypes will then be used to solicit input from the software advisory committee and the general user community for finalization of the design.

During CY 2002, Montana State University (MSU) and the Idaho National Engineering and Environmental Laboratory (INEEL) have also completed the initial development of the source specification module for the targeted radionuclide modality. Finally, the software requirement specification has been refined and posted on the MSU web page for the project, http://boron.csnet.montana.edu.

\section{PATIENT MODULE}

One of the principal responsibilities of the Patient module is creating and managing the database connection. During CY 2002, a new class, DatabaseToolbox, was created to more efficiently communicate with the database. The Patient module was updated so that it now incorporates the new DatabaseToolbox class. An improved interface was created to make the connection with the database more visible. The user now has the ability to select different databases. If the Patient module is unable to complete the database connection, the program can still run and provide diagnostics to the user.

\section{IMAGE MODULE}

The Image module prepares and processes patient images for use by the other MINERVA modules. During CY 2002, database connectivity was added to the Image module. The Image module, with the appropriate plugin, can convert externally formatted images to UnivelScans (the internal MINERVA image format) that can be made available to all of the other modules through the database. Plugin support was also added so that operations and image import filters 
can be added later by placing a plugin in the plugins folder. Currently, there is only one image import plugin that reads QSH files.

The image registration system has been fully converted to Java, and is undergoing testing and performance improvement. The registration system currently implements the minimization of mutual information using a parabolic optimizer with Brent's method. ${ }^{2}$ Brent's method accelerates a parabolic search by attempting to directly solve for the minimum based on the current threepoint solution rather than waiting for convergence. This procedure can dramatically improve search times for smooth functions.

Finally, several utility classes were created to support the Image Module, including the Preferences class for managing user preferences, the PluginManager class for managing plugins to the system, and the MINERVAIcons class.

\section{MODEL MODULE}

The Model module creates the anatomical geometry of the patient and target based on the image data. The Model module is used to either create a new univel ${ }^{3}$ geometry file or edit an existing model.

During CY 2002 several changes were made. First, the number of region identifiers was increased from 256 to 65,536. A generic undo manager was added that allows the program to record the actions the user, and later undo those actions. A single image view was added, so that the user could see and operate on one image slice at a time. Zooming support was also implemented. The display of image slices was also optimized for speed and memory usage by doing away with unnecessary caching, and displaying the image slices directly from the data structure rather than first copying it to a temporary buffer. Finally, the model module was updated to use the new DatabaseToolbox and PatientData classes.

\section{PLAN AND REPORT MODULES}

The division of labor between the Plan and Report modules was finalized during CY 2002. The Plan module will now control the launching of the source and transport plugins, receive the results from these plugin modules, and save the results to the database. The dose data from the transport modules will, if necessary, be interpolated onto the univel grid before storage in the database. Plans, which are defined as a weighted combination of one or more fields, are specified in this module, but the dose combination is performed in the Report module, so that Relative Biological Effectiveness (RBE) factors and Neutron Capture Therapy (NCT) agent concentrations may be included in the weighting.

The Report module will access the database to read the dose information for each field in the plan, and perform the dose combination by weighting the field dose arrays by the specified RBE factors and NCT agent concentrations. This step is performed in the Report module, instead of the Plan module, to allow the user to alter the RBE factors and NCT agent concentrations as part of the plan analysis step. A widget will be available to allow the user to define RBE factors by 
field, region, and dose component. In addition, the Report module will designate one or more plans to be used for patient treatment.

Progress on the Report module during CY 2002 included definition of the various plugin functions to calculate several types of dose-volume histograms. Development of the user interface was started, and the methods for accessing the dose information from the patient database were developed.

\section{DATABASE}

The DatabaseToolbox Class was developed with the following objectives: (1) initialize a new database for use with MINERVA, (2) setup the database connection between MINERVA modules and the database, (3) allow other developers to access the database without knowledge of the Java Database Connectivity (JDBC ${ }^{4}$ ) and the Application Programming Interface (API), and (4) abstract any database specific implementations (BLOB support, transactional tables, etc.).

Some of the fundamental database operations have been changed since last year's report. The previous operations required that the DatabaseToolbox have complete knowledge of the data class representation in the database. This introduced unnecessary complexities when it became necessary to change the contents of the data classes, as these changes must be communicated to the DatabaseToolbox class. This also lead to a rather large DatabaseToolbox class. The new DatabaseToolbox has been generalized so that it does not need to know how the data class should be stored ahead of time. Only the data classes need to provide that information when they are saved or loaded from the database. If there are any changes made to the data classes, the changes are all contained in the data class, and nothing needs to be changed in the DatabaseToolbox.

The way the data classes write to the database has also been generalized. They no longer make direct calls to the DatabaseToolbox. The DataStore class now mediates between the two, first asking the data class for information and then forwarding it to the DatabaseToolbox. This can also be used for the $\mathrm{XML}^{5}$ output, because the DataStore could just as easily forward the information to a XMLToolbox rather than the DatabaseToolbox.

\section{FILE IMPORT/EXPORT FEATURES}

Because database files are generally hidden from the user and not easily transportable, a convenient way to transfer patient information between various users and installations must be developed. To accomplish this, a complete XML file specification of all of the information contained in the database and a set of XML-based import/export tools are being developed. This same XML file specification will also be used to define the API between MINERVA and the modality specific transport modules.

The XML file specification is put into a verifiable and standard XML document using the XML Schema. To do this, Apache Xerces is used to parse XML documents, and the Java Document Object Module (JDOM) is used to manipulate them internally. 
The XML-based tools: (1) allow selected "branches" of a patient XML file to be imported into the database, (2) allow a patient (or partial patient) to be exported to an XML file, (3) write XML for transport modules and read the resulting XML file back into the database, and (4) write XML for source modules and read the resulting XML back into the database. During CY 2002, a large part of the MINERVA XML file specification was developed and verified.

Figure 1 provides an overview of the MINERVA data flow.

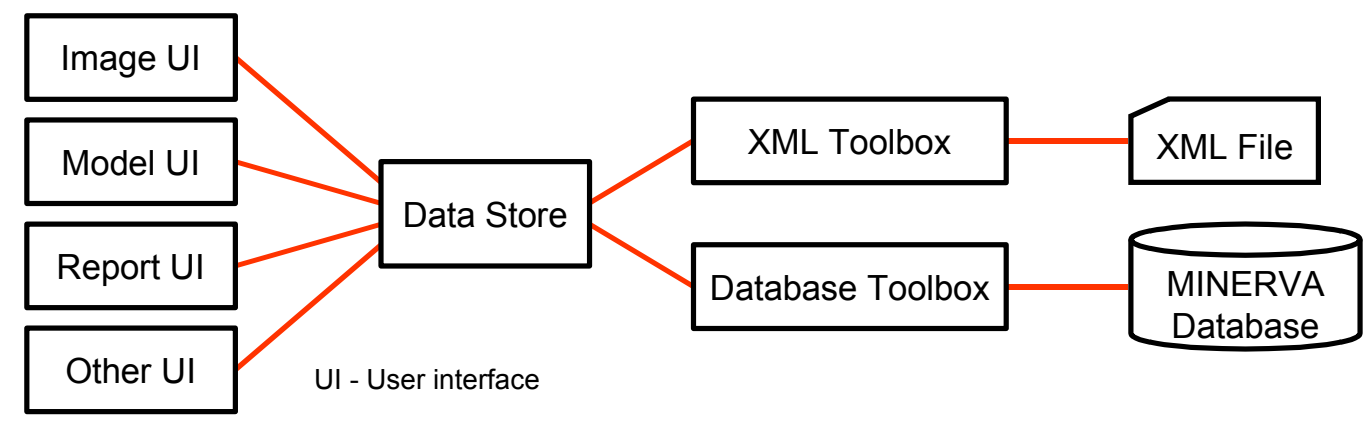

Figure 2. MINERVA data flow design.

\section{PLUGIN MANAGER}

The plugin manager provides a way to expand MINERVA after it has been distributed, without needing to change the actual application. The user adds new plugins into a plugin folder, and the capabilities in those plugins will be loaded into MINERVA without any need to change the MINERVA application. Plugins also provide a way to break problems into small parts that can be independently created. For example, the image module needs to import images from a variety of sources. For each source, a plugin can be made that will show up in the image module's import menu.

Each plugin is contained within a Java Archive File (JAR), which is a collection of files that have been combined into one file. This allows a plugin to combine many classes, icons, help files, and any other resources that might be needed, into one file. Having just one file makes it much easier to distribute the plugin. It is also easier for end users to install one file than many different files. JAR files also have the advantage of being compressed, so that they take up less space on disk, and take less time to distribute.

\section{TARGETED RADIONUCLIDE SOURCE SPECIFICATION AND TRANSPORT MODULES}

Development commenced on the targeted radionuclide source specification module. Using the patient geometry and the emission image (an image created by taking whole body gamma camera radiation scans of the patient after a test dose of the radionuclide has been administered), the module determines the activity distribution, on a univel basis, necessary to reproduce the emission image. Backprojection methods were used to account for tissue attenuation between 
the source univel and the detector array. This activity distribution can then be used as a source for the targeted radionuclide transport module to determine the three dimensional dose distributions received by the patient.

As part of this development effort, the univel tracking methods from the previous code SERA ${ }^{6}$ (Simulation Environment for Radiotherapy Applications) (seraMC) have been converted to the Java language and tested for accuracy and performance. The Java code is about 2.5 times slower than optimized $\mathrm{C}$, and during the next year, strategies will be developed to narrow this gap, as performance is a critical issue for the transport module.

\section{CALCULATION ENGINE VERIFICATION FOR MOLECULAR TARGETED RADIOTHERAPY}

The Lawrence Livermore National Laboratory (LLNL) Peregrine Monte Carlo code system ${ }^{7}$ will be used as the calculation engine for targeted radionuclide therapy in MINERVA. The code, originally written for and applied to external beam therapy, has been extended to now include internal sources. For this purpose, it accepts a three dimensional activity map of the patient as an input with additional information about the isotope used. It simulates the resulting dose distribution in the patient.

The extended code has been thoroughly tested in comparison with published results of other Monte Carlo codes (EGS4 ${ }^{8}$ and $\mathrm{MCNP}^{9}$ ) and the Medical Internal Radiation Dose (MIRD) system, a dosimetry standard in Nuclear Medicine. Specifically, the comparison with the other codes concentrated on the calculation of absorbed fractions in spheres of radioactive material. Spheres of a comprehensive set of different radii have been investigated for monoenergetic photon and electron sources, and for radioisotope photon emission. The results have been published in a peer-reviewed journal. ${ }^{10,11}$ MINERVA prototype simulations, using an initial software prototype system established for proof of principle, agree well with the other Monte Carlo codes. They are generally within 2\% of EGS4 and 10\% of MCNP (Figures 2-3).

However, the MINERVA results differ by up to $40 \%$ from the recommendations given in MIRD pamphlets 3 and 8 for identical medium composition and density. For several representative source and target organs in the abdomen and thorax, specific absorbed fractions calculated with the MINERVA system are generally within 5\% of those published in the revised MIRD Pamphlet 5 for $100 \mathrm{keV}$ photons. However, results differ by up to $23 \%$ for the adrenals, the smallest of the target organs. Table 1 shows the comparison of specific absorbed fractions calculated with the MINERVA prototype code and the specific absorbed fractions given in revised MIRD pamphlet. 


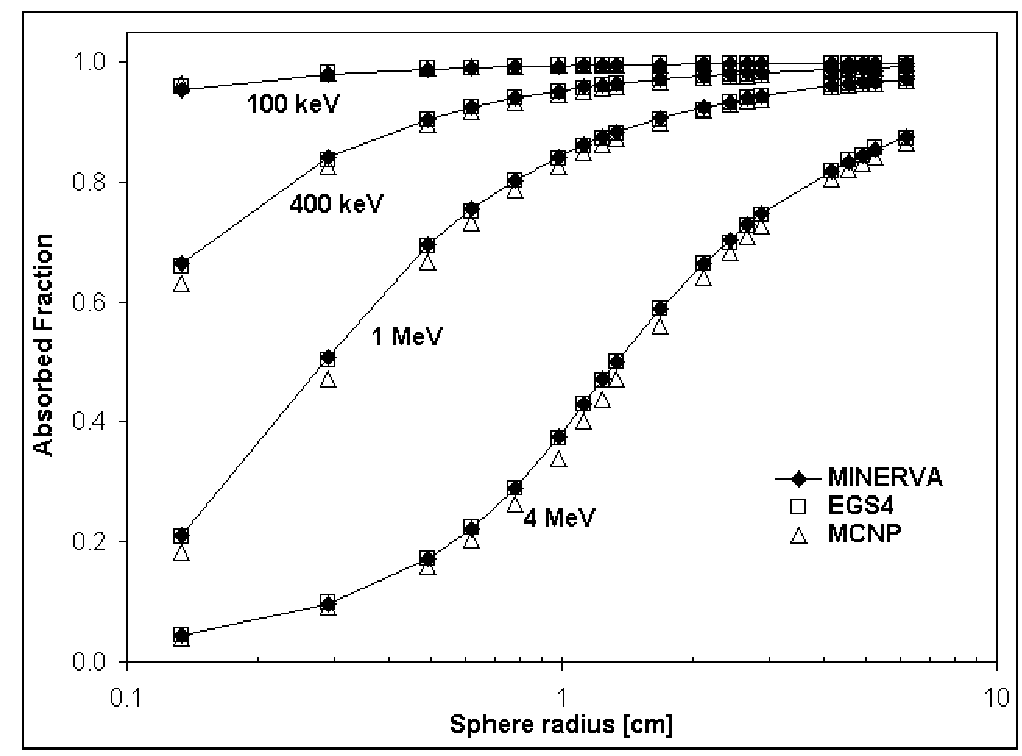

Figure 2. Comparison between absorbed fractions for MINERVA prototype, EGS4 and MCNP for $100 \mathrm{keV}, 400 \mathrm{keV}, 1 \mathrm{MeV}$, and $4 \mathrm{MeV}$ electron spherical sources. Similar to photons, MINERVA and EGS4 are in good agreement, but are as much as $10 \%$ higher than MCNP. (Figure from Descalle, et al. ${ }^{11}$ )

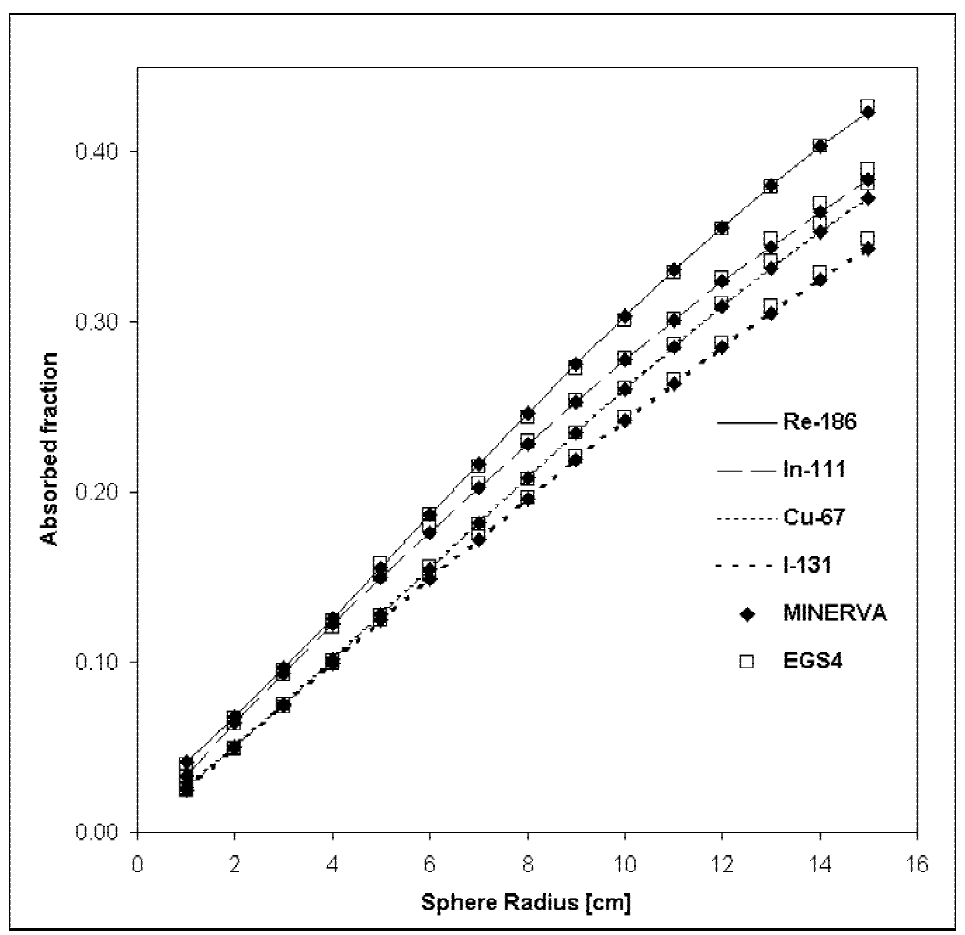

Figure 3. Comparison between MINERVA prototype and EGS4 prediction of absorbed fractions for several spherical sources with radioisotopes of interest: emitted photons and all daughter particles are tracked. MINERVA and EGS4 agree to within $2 \%$. (Figure from Descalle, et al. ${ }^{11}$ ). 
Table 1. Comparison of specific absorbed fractions calculated with the MINERVA prototype code and the specific absorbed fractions given in revised MIRD pamphlet $5 \mathrm{f}$ for $100 \mathrm{keV}$ photons emitted by source organs of a heterogeneous phantom. Source organs are the adrenals, the kidneys and the liver; and target organs are the lungs, adrenals, kidneys, liver, pancreas and spleen. (Table from Descalle, et al. ${ }^{11}$ ).

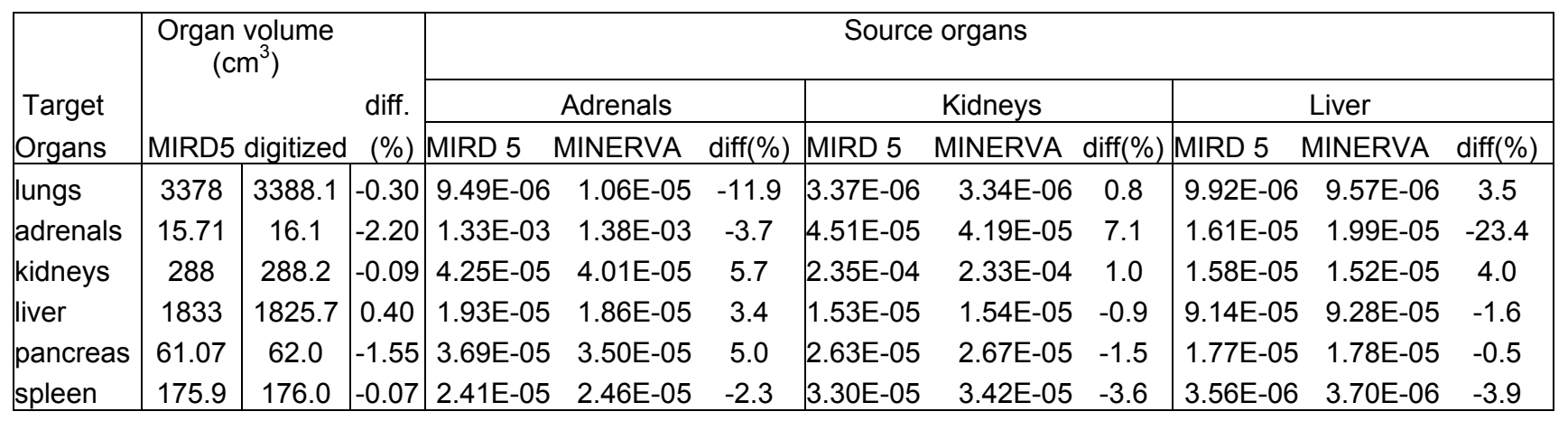

Absorbed dose distributions have been obtained in a patient's anatomy by assigning radionuclide concentration to the kidney in two different patterns. The three dimensional (3D) representation of the patient's anatomy was defined by computed tomography (CT) scan slices extending from the thorax to the pelvis. The 3D radiation source was generated as follows: the right kidney was identified and defined a region of interest (ROI) on the appropriate CT scans, assigned a homogeneous activity inside the ROI, and a source image was generated for each CT scan containing the ROI. There is a one-to-one correspondence between CT scan and source-image pixels. Three radionuclides were considered: ${ }^{131} \mathrm{I},{ }^{67} \mathrm{Cu}$, and ${ }^{90} \mathrm{Y}$. Average beta and photon energies are $0.183 \mathrm{MeV}$ and $0.382 \mathrm{MeV}$ for ${ }^{131} \mathrm{I}$, and $0.142 \mathrm{MeV}$ and $0.115 \mathrm{MeV}$ for ${ }^{67} \mathrm{Cu} .{ }^{90} \mathrm{Y}$ was assumed to emit only betas with an average energy of $0.934 \mathrm{MeV}$. Figures 4 and 5 show a transverse axial cut through the resulting radiation absorbed dose distribution, overlaid on the patient's CT scan. Figure 4 demonstrates the effect of a homogenous distribution of ${ }^{131} \mathrm{I}$ in a patient's right kidney. The activity distribution is shown in the left image, the resulting absorbed dose in the right image. The radiation absorbed dose inside the kidney is primarily caused by beta emissions, while dose outside the kidney is caused by emitted gamma rays. 


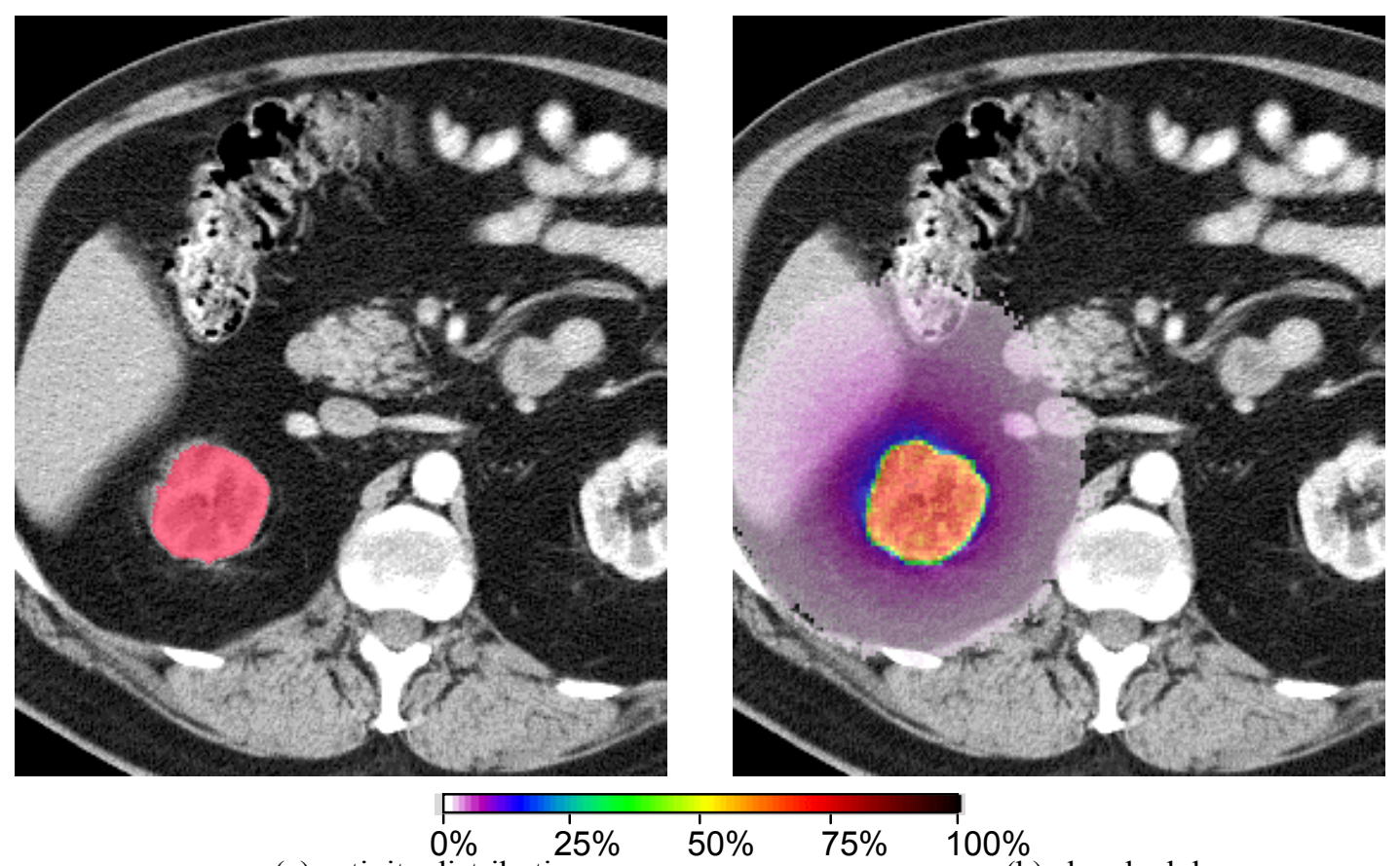

(a) activity distribution

(b) absorbed dose

Figure 4: Dose distribution of ${ }^{131} \mathrm{I}$ in a kidney obtained with 3D Monte Carlo simulations.

The patient's anatomy, as determined from the CT scan, was used to outline the kidney volume and define the Monte Carlo transport grid. In Figure 4, the left image (a) shows an axial slice through the uniform activity distribution of ${ }^{131}$ I assigned to the right kidney. The resulting radiation absorbed dose distribution is shown on the right (b). Relative radiation absorbed dose is represented in a rainbow spectrum with red representing high dose and purple showing low dose.

Figure 5 shows the consequences of uniform activity distributions of ${ }^{131} \mathrm{I},{ }^{67} \mathrm{Cu}$, and ${ }^{90} \mathrm{Y}$ confined to the cortex of the kidney. Colors vary linearly with dose from red (greatest relative intensity), through yellow, green, blue, and purple to white (least relative intensity). For all three isotopes, significant dose heterogeneities are noticeable within the kidney. These images also illustrate the difference between ${ }^{90} \mathrm{Y}$ and the other two isotopes. Unlike ${ }^{131} \mathrm{I}$ and ${ }^{67} \mathrm{Cu},{ }^{90} \mathrm{Y}$ emits only betas (electrons), and since electrons have a fixed, limited range in tissue, there is almost no dose outside the kidney resulting from the activity within the organ, whilst the dose from Bremsstrahung photons that escape the kidney is less than $1 \%$ of the maximum dose at distances greater than $1.1 \mathrm{~cm}$, the electron maximum CSDA range for ${ }^{90} \mathrm{Y}$ maximum beta emission at 2.28 $\mathrm{MeV}$. 

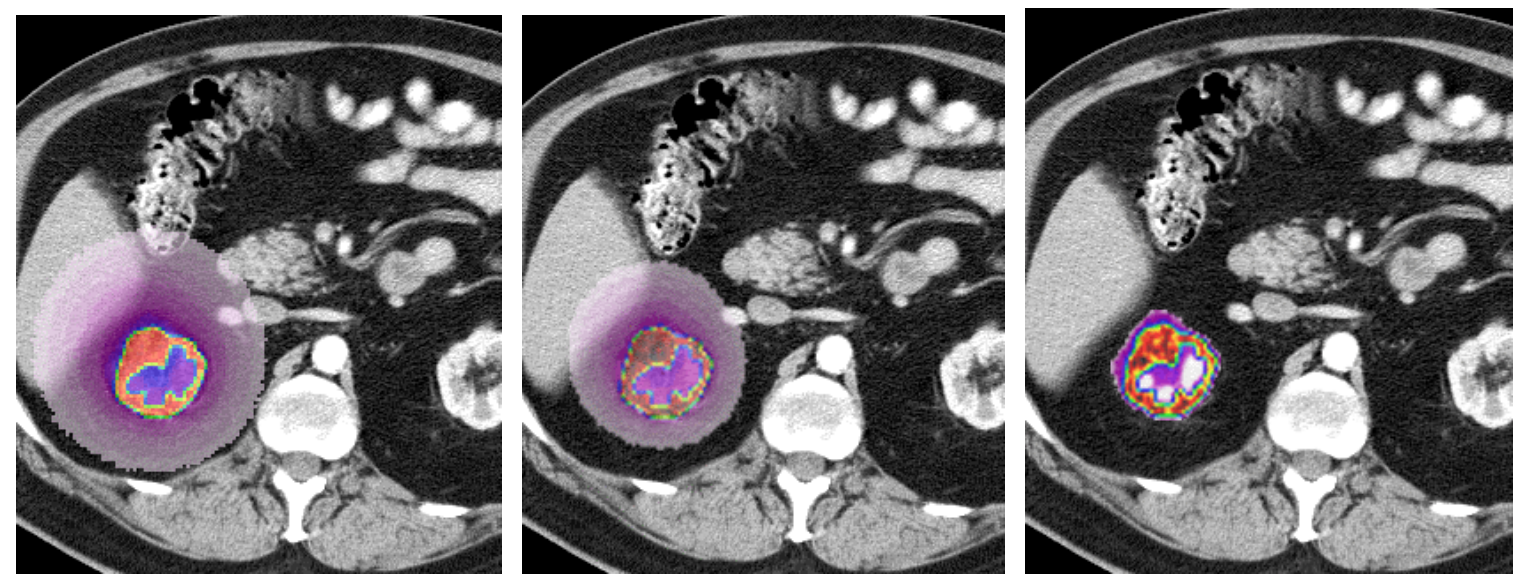

(a) ${ }^{131} \mathrm{I}$

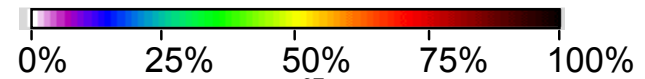

(b) ${ }^{67} \mathrm{Cu}$

(c) ${ }^{90} \mathrm{Y}$

Figure 5. Dose distribution of ${ }^{131} \mathrm{I},{ }^{67} \mathrm{Cu}$, and ${ }^{90} \mathrm{Y}$ in a kidney obtained with 3D Monte Carlo simulations.

The transport geometry is defined by a 3D set of CT scans. In Figure 5, the right kidney was selected as the source organ, and a uniform activity distribution was assigned to the cortex of the right kidney. There is no activity assigned to the rest of the kidney. A transverse axial slice of the absorbed radiation dose distribution is shown overlaid on the relevant CT slice of the patient. The resulting dose distributions are shown for three isotopes: ${ }^{131} \mathrm{I}$ (a), ${ }^{67} \mathrm{Cu}$ (b) and ${ }^{90} \mathrm{Y}$ (c). Relative radiation absorbed dose is represented in a rainbow spectrum with red representing high dose and purple showing low dose. Each calculation is self-normalized with red representing the highest dose region.

\section{PEREGRINE MOLECULAR TARGETED RADIOTHERAPY CALCULATION ENGINE PLUGIN}

Development commenced on the link between the API and the Peregrine calculation system. The Peregrine input/output $(\mathrm{I} / \mathrm{O})$ is to be adapted through a software interface to connect into the API. The software will read the MINERVA markup language (MML) data and transmit the necessary information to the Peregrine I/O for the simulation. Following the simulation, the software will read the result from Peregrine and update the MML file to be sent back to the API. At this point, MML files can be read. Information, including images, can also be inserted into the MML file. A set of Peregrine XML tools to interact with the Peregrine I/O are being worked on to complete the interface.

\section{SCIENTIFIC DOSIMETRY EFFORT FOR MOLECULAR TARGETED RADIOTHERAPY}

The importance of treatment planning and accurate dosimetry for molecular targeted radiotherapy was established through argumentation in the paper entitled "Treatment planning for molecular targeted radionuclide therapy," published in the journal Cancer Biotherapy and 
Radiopharmaceuticals. ${ }^{12}$ This comprehensive overview illustrates the necessity of accurate dosimetry using applicable tumor control probabilities (TCP) and Normal Tissue Complication Probabilities (NTCP) determined with external radiation therapy. A new quantity, effective tumor control probability (eTCP), is introduced to enable direct connection and comparison of TCP and NTCP and demonstrate the dose effect relationship in molecular targeted radiotherapy.

The importance of accounting for change in mass, particularly tumor regression, when assessing absorbed radiation dose in radioimmunotherapy (RIT) for non-Hodgkin's lymphoma for tissues whose mass changes during the time the radiation dose is being absorbed, has also been shown. The increase in calculated absorbed dose when this change is considered provides better insight into the high nodal response rates observed in non-Hodgkin's lymphoma patients. ${ }^{13}$

These dose calculation algorithms were also used in other radioimmunotherapy studies and helped show that, if bone marrow support is adequate, the radiation dose historically tolerated by normal organs other than marrow would allow thirty percent increase in the administered dose, resulting in a mean dose of 95 Gy to metastatic prostate cancer using DOTA-Peptides. ${ }^{14}$ Again, precise dose calculation is essential.

Furthermore, the calculation algorithms were applied to show that Cathepsin-degradable peptides used to link chelated radiometals to antibodies reduce liver radiation dose and improve the therapeutic index (TI) for RIT given in combination with bone marrow support. ${ }^{15}$

The calculation algorithms were used to investigate the impact of interpatient pharmacokinetic variability on design considerations for therapy with radiolabeled monoclonal antibodies. ${ }^{16}$

\section{REFERENCES}

1. INEEL Advanced Radiotherapy Research Program Annual Report 2001, J.R. Venhuizen (ed), INEEL/EXT-02-00060, April 2002, pp 51-66.

2. Cambridge Press, "Numerical Recipes in C," Cambridge Press, 1992, pp 402.

3. M.W. Frandsen, D.E. Wessol, F.J. Wheeler, D. Starkey, "Rapid Geometry Interrogation for Uniform Volume Element-Based BNCT Monte Carlo Particle Transport Simulation," Proceedings of the Eighth International Symposium on Neutron Capture Therapy, September 13-18, 1998, La Jolla, CA, Plenum Press, New York.

4. Java Database Connectivity - http://java.sun.com/products/jdbc/overview.html.

5. Coding of multimedia and hypermedia information -- Part 8: XML notation for ISO/IEC 13522-5.

6. D.W. Nigg, C.A. Wemple, D.E. Wessol, F.J. Wheeler, "SERA-An Advanced Treatment Planning System for Neutron Therapy and BNCT," Trans. ANS, 80:66-68, 1999. 
7. C.L. Hartmann Siantar, R.S. Walling, T.P. Daly, B. Faddegon, N. Albright, P. Bergstrom, A.F. Bielajew, C. Chuang, D. Garrett, R.K. House, D. Knapp, D.J. Wieczorek, L.J. Verhey, "Description and dosimetric verification of the PEREGRINE Monte Carlo dose calculation system for photon beams incident on a water phantom," Med Phys, 28(7). 1322-37, 2001.

8. W.R. Nelson, H. Hirayama, D.W.O. Rogers, The EGS4 Code System, Stanford Linear Accelerator Center Report SLAC-265, Stanford CA., 1985.

9. J.F. Briesmeister, MCNP - A General Monte Carlo N-Particle Transport Code, Version 4A, LA-12625-M, Los Alamos National Laboratory, 1993.

10. G.L. DeNardo, C.L. Hartmann Siantar, S.J. DeNardo, "Radiation dosimetry for radionuclide therapy in a nonmyeloablative strategy," Cancer Biotherapy and Radiopharmaceuticals, 17(1) $107-118,2002$.

11. M.A. Descalle, C.L. Hartmann Siantar, L. Dauffy, D.W. Nigg, C.A. Wemple, A. Yuan, G.L. DeNardo, "Application of MINERVA Monte Carlo simulations to targeted radionuclide therapy," Cancer Biotherapy and Radiopharmaceuticals, 18(1). 71-80, 2003.

12. C.L. Hartmann Siantar, K. Vetter, G.L. DeNardo, S.J. DeNardo "Treatment planning for molecular targeted radionuclide therapy," Cancer Biotherapy and Radiopharmaceuticals, 17(3) 267-280, 2002.

13. C.L. Hartmann Siantar, G.L. DeNardo, S.J. DeNardo, "Impact of Nodal Regression on Radiation Dose for Lymphoma Patients following Radioimmunotherapy," in press, Journal of Nuclear Medicine.

14. S.J. DeNardo, et al., "Enhanced Therapeutic Index of Radioimmunotherapy in Prostate Cancer Patients: Comparison of Radiation Dosimetry for DOTA-Peptide Versus 2IT-DOTA MAb Linkage for RIT," submitted to Clinical Cancer Research.

15. G.L. DeNardo, et al., "Preclinical Evaluation of Cathepsin-degradable Peptide Linkers for Radioimmunoconjugates," submitted to Clinical Cancer Research.

16. G.L. DeNardo, A. Yuan, D. Goldstein, C. Richman, R. O'Donnell, S. Shen, C.L. Hartmann Siantar, S.J. DeNardo, "Impact of interpatient pharmacokinetic variability on design considerations for therapy with radiolabeled MAbs," in press, Cancer Biotherapy and Radiopharmaceuticals. 


\title{
NEUTRONIC PERFORMANCE ASSESSMENT OF AN EPITHERMAL NEUTRON BEAM FOR PRECLINICAL BNCT RESEARCH AT WASHINGTON STATE UNIVERSITY
}

\author{
D.W. Nigg ${ }^{1}$, C.A. Wemple ${ }^{1}$, J.R. Venhuizen ${ }^{1}$, J.K. Hartwell ${ }^{1}$, G.E. Tripard ${ }^{2}$, S. Sharp ${ }^{2}$, K. \\ Fox $^{2}$, and P.R. Gavin ${ }^{2}$
}

1. Idaho National Engineering and Environmental Laboratory, Idaho Falls, ID

2. Washington State University, Pullman, WA

\section{INTRODUCTION}

Radiation oncology researchers at the Washington State University (WSU) School of Veterinary Medicine have made major contributions over the years to the understanding of the in-vivo radiobiology of Boron Neutron Capture Therapy (BNCT). For example, the large-animal model studies of normal brain tissue tolerance in BNCT conducted by this group ${ }^{1}$ provided a key component of the radiobiological basis for the resumption of human BNCT trials in the U.S. in 1994. Those preclinical studies used the epithermal-neutron beams available at Brookhaven National Laboratory (no longer available) and at the Petten facility, in The Netherlands, with technical support from the Idaho National Engineering and Environmental Laboratory (INEEL) in several areas of physics, biophysics, and analytical chemistry. More recently, attention has been focused upon the construction of a more convenient and cost-effective, local epithermalneutron beam facility at WSU for collaborative INEEL/WSU BNCT preclinical research and boronated pharmaceutical screening in cell and animal models. The design ${ }^{2,3}$ and construction ${ }^{4,5}$ of the new facility, installed in the thermal column region of the TRIGA ${ }^{\text {a }}$ research reactor at WSU, was performed in a collaborative effort of WSU and the INEEL. This article summarizes the results of neutronic performance measurements for the WSU beam that were completed during calendar year 2002 .

\section{FACILITY DESCRIPTION}

The $1 \mathrm{MW}$ WSU reactor core is suspended from a movable bridge above the pool as shown in Figure 1. It can be positioned directly adjacent to a truncated aluminum cone that extends horizontally into the reactor pool from the tank wall on the upstream side of the filtering and moderating assembly. This cone and the adjacent thermal column area were originally filled with graphite, comprising the thermal neutron column (or thermal-column) for the WSU Nuclear Radiation Center. A water-filled shield was located downstream of the graphite thermal column. There was a large open floor area outside of this final shield.

Figure 2 shows a schematic diagram of the WSU epithermal-neutron beam facility. The new epithermal-neutron beam extraction components are located in the thermal-column region of the reactor-shielding monolith. The original graphite has been removed from this region and replaced with a new epithermal-neutron filtering, moderating, and collimating assembly as

\footnotetext{
${ }^{a}$ TRIGA is a trademark of General Atomics of San Diego, CA.
} 
shown. Neutrons emanating from the core travel into the filtering and moderating region. The spectrum is tailored in this region such that most neutrons emerge with energies in the epithermal energy range $(0.5 \mathrm{eV}-10 \mathrm{keV})$. Downstream of the filtering and moderating region is a bismuth and lead gamma shield, followed by a conical neutron collimator composed of bismuth, surrounded by borated polyethylene. Provision is made for several different exit port aperture sizes as shown. A heavily shielded concrete beam stop and treatment room has been constructed just outside of the thermal column opening in the reactor shield wall, as shown in Figure 3.

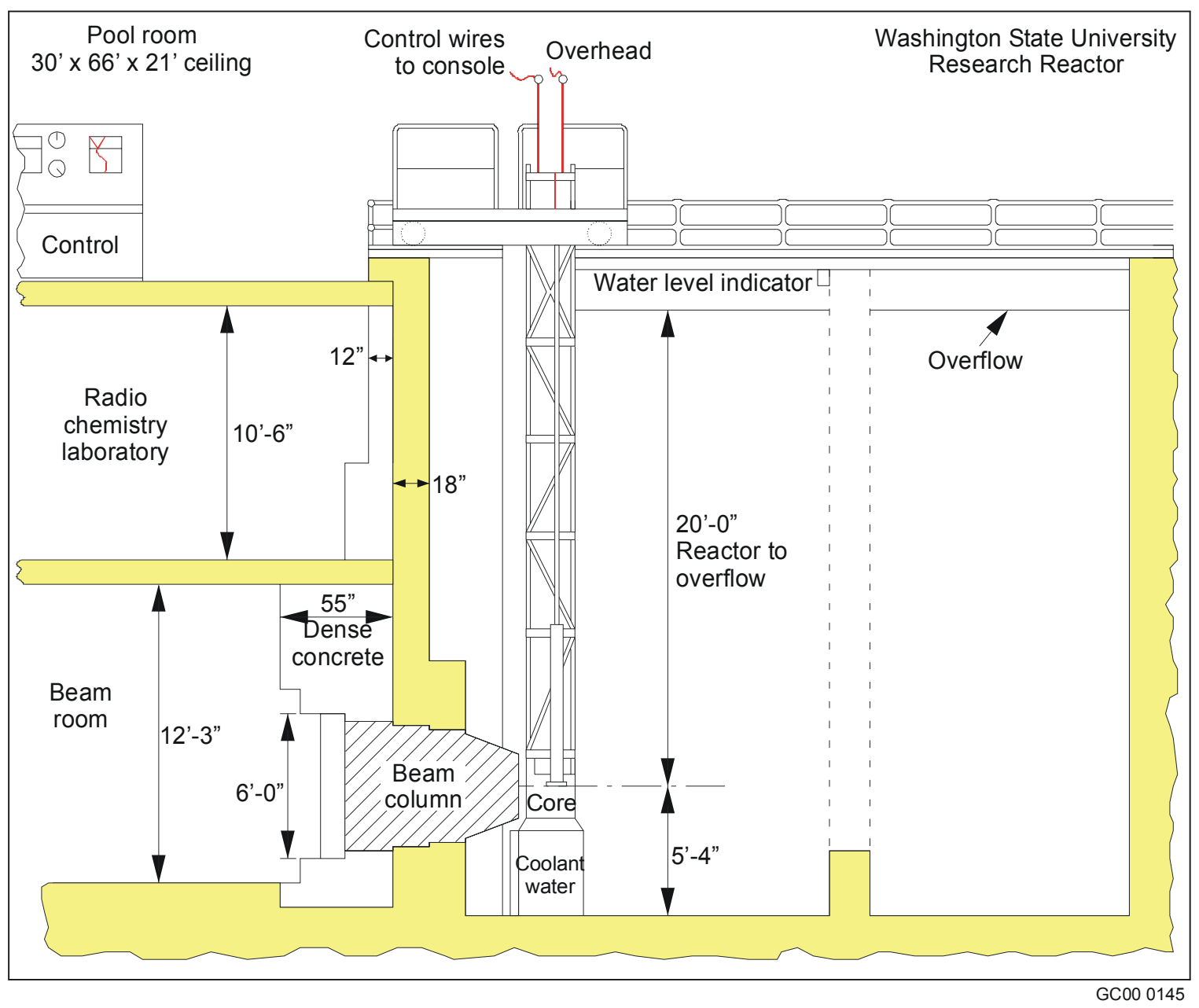

Figure 1. Elevation plan of the WSU TRIGA reactor facility. 


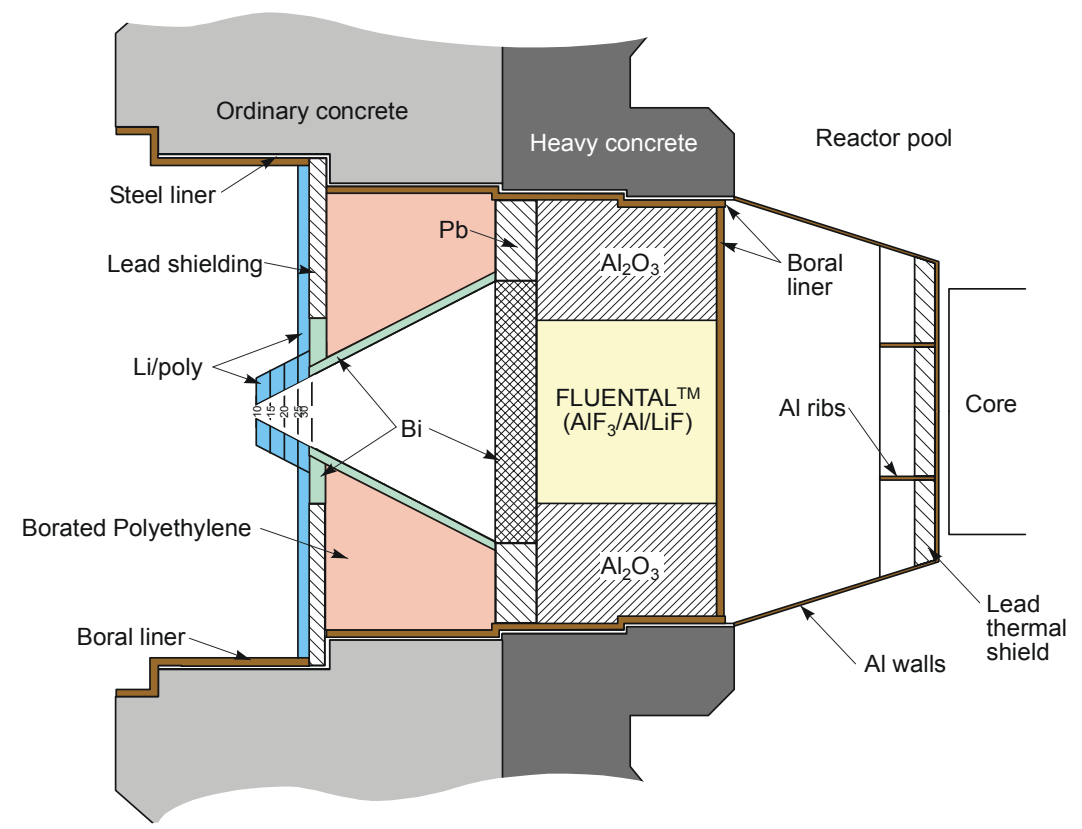

Not to scale

Figure 2. WSU column assembly, with epithermal neutron filter in place.

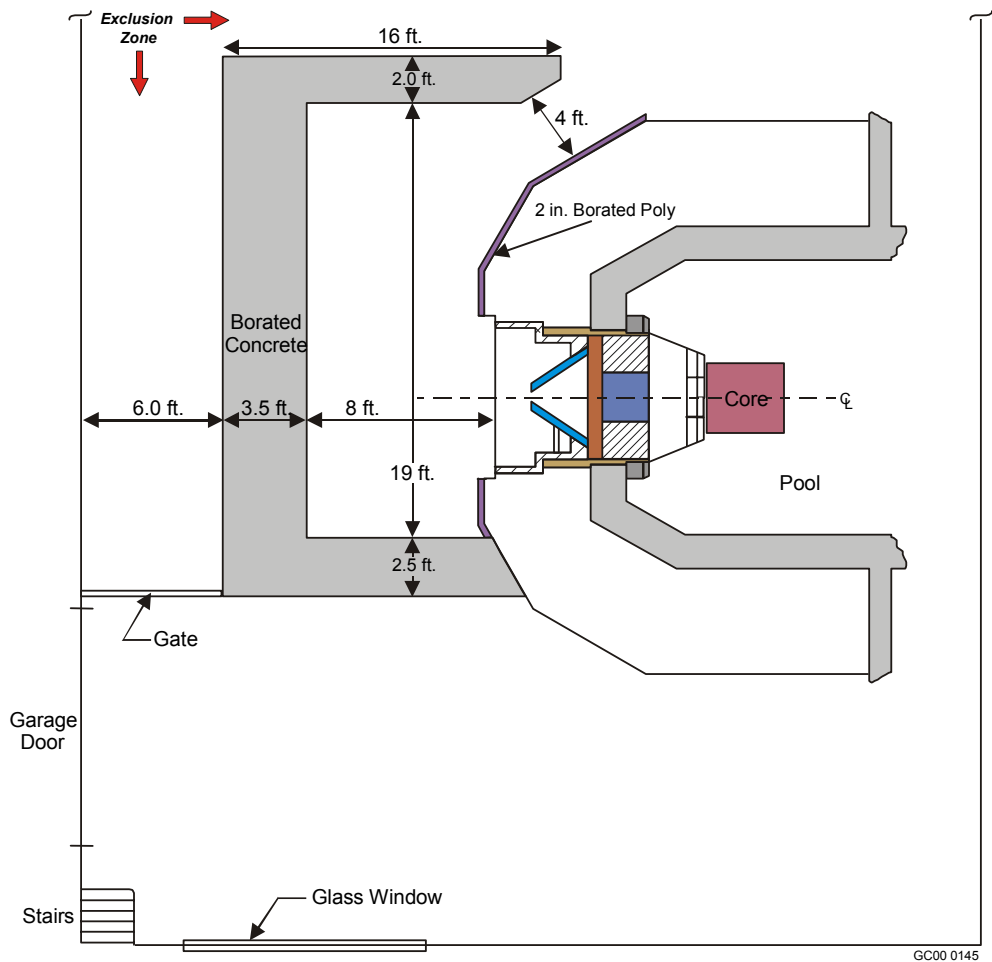

Figure 3. Approximate WSU beam stop and treatment room arrangement. 
A key distinguishing feature of the WSU facility is the incorporation into the design of a new, high-efficiency, neutron moderating and filtering material (FLUENTAL) ${ }^{\mathrm{b}}$ developed by the Technical Research Centre of Finland. ${ }^{6}$ FLUENTAL is manufactured by hot isostatic pressing of a mixture of $69 \%$ (by weight) aluminum fluoride, $30 \%$ aluminum, and $1 \%$ lithium fluoride. A block of this material, having a thickness in the beam propagation direction of $0.64 \mathrm{~m}$ and transverse dimensions of approximately $0.6 \mathrm{~m}$, is surrounded by aluminum oxide to produce the neutron filtering and moderating region, as shown in Figure 2. $\mathrm{MCNP}^{7}$ and $\mathrm{DORT}^{8}$ radiation transport design calculations for the coupled core and filter-collimator assembly indicate that a free-beam epithermal neutron flux of approximately $10^{9} \mathrm{n} / \mathrm{cm}^{2}$-s at a reactor power of $1 \mathrm{MW}$ will be produced at the exit port of the collimator (with the reactor core optimally loaded). The background neutron KERMA rate per unit useful epithermal neutron flux (a measure of the fastneutron contamination) for the beam is calculated to be approximately $3.0 \times 10^{-11} \mathrm{cGy} / \mathrm{n}-\mathrm{cm}^{2}$. The computational methods used for this design were previously validated against INEEL measurements performed for a similar neutron beam facility that is already in operation at the FiR1 TRIGA research reactor in Finland. ${ }^{9}$

An additional key feature of the WSU beam facility design is the provision for adjustable filtermoderator thickness to permit systematic exploration of the radiobiological consequences of increasing the fast-neutron contamination above the nominal value associated with the baseline system described above. This is an important clinical issue for BNCT. Thinner filter/moderator arrangements will produce neutron beams having correspondingly harder spectra and greater levels of fast-neutron contamination. The beamline components shown in Figure 2 are designed for relative ease of disassembly and re-assembly compared to other reactor-based epithermalneutron facilities that are currently in operation. Thus, it will be possible to have a number of different filter/moderator arrangements over the life of the facility.

Figure 4 shows the collimator installation. The collimator, assembled from 16 identical bismuth "barrel-stave" castings, is a truncated right circular cone with a base opening diameter of $91.4 \mathrm{~cm}$ (36 in.), a height of $38.1 \mathrm{~cm}$ (15 in.), and a wall thickness of $3 \mathrm{~cm}(1.2 \mathrm{in}$.$) . The angle of the$ cone is 45 degrees. It is supported by borated polyethylene and two rows of lead bricks, as shown in Figure $4 \mathrm{~b}$. The downstream end of the collimator is fitted with a bismuth collar designed to provide a transition from the circular shape of the cone to the square shape required to match the lead shielding bricks in the final shield wall. The collar will accommodate borated or lithiated polyethylene inserts having various beam port aperture sizes and field shaping configurations, as shown in Figure 2. Finally, Figure 5 shows the positioning of the final lithiated polyethylene neutron suppression shield on the outer surface of the bismuth-lead shield wall.

\footnotetext{
${ }^{\mathrm{b}}$ FLUENTAL is a trademark of the Technical Research Center of Finland.
} 


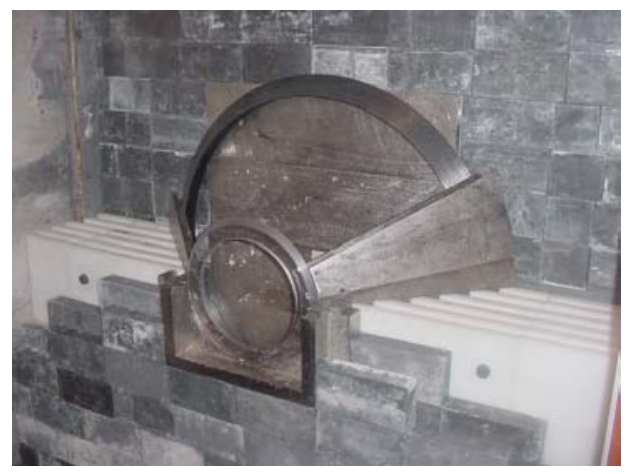

(a)

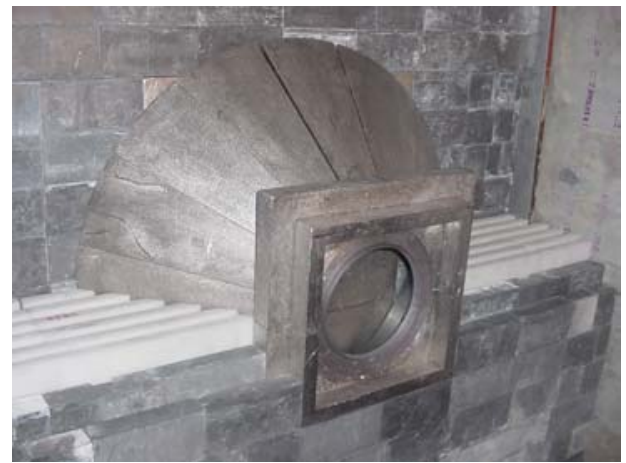

(b)

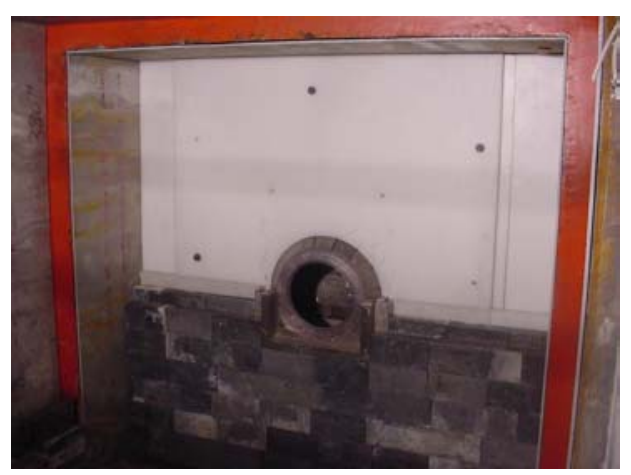

(c)

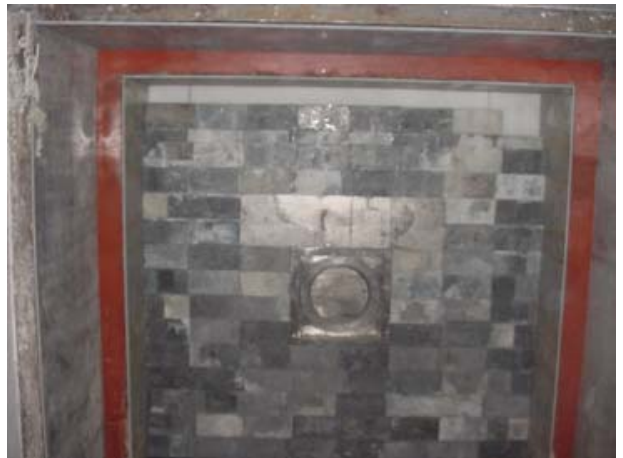

(d)

Figure 4. Final beam collimator installation at WSU showing (a) the partially installed collimator, (b) the fully installed collimator with square exit port collar and lower borated polyethylene shielding, (c) the upper borated polyethylene shielding, and (d) the final lead shielding.

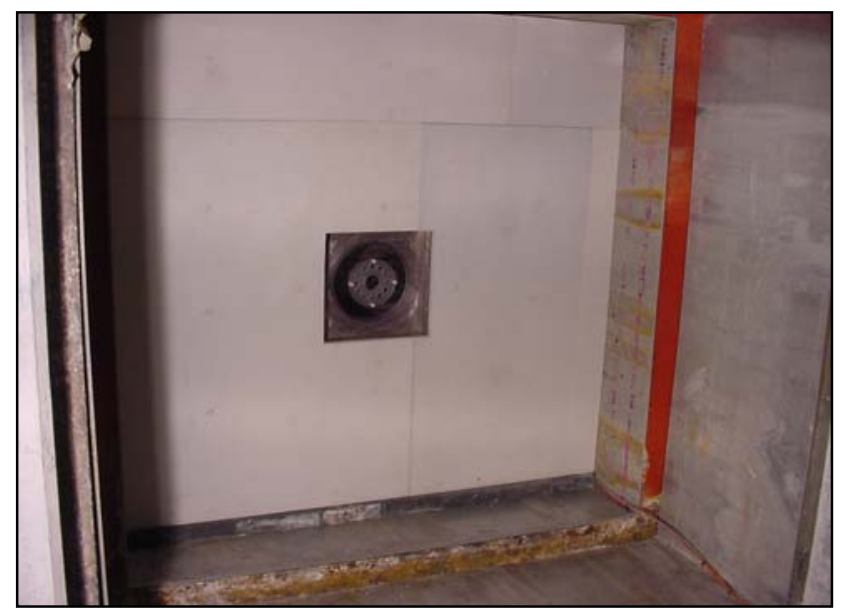

Figure 5. Lithiated polyethylene neutron suppression shield placement. 


\section{METHODS AND MATERIALS}

Measurements of the free beam spectrum, as well as some in-phantom beam performance measurements, were completed during the year. The various experimental methods are described in the following sections.

Activation Foil Measurements in the Source Plane. The first free beam spectrum measurements were focused on characterization of the neutron spectrum in an imaginary transverse "source plane" passing through the base of the square flange on the downstream side of the bismuth collar. The source plane is a mathematical construct used for specifying the neutron source boundary conditions for the various dosimetry and treatment planning computations that will be required to support each experimental irradiation at WSU. By definition, the physical beamline components upstream of the source plane do not change from one irradiation to the next. Components downstream of this plane, such as the previously mentioned lithiated polyethylene field shaping plates, can change for each irradiation, and are therefore explicitly modeled in dosimetry and treatment planning computations. Time-consuming beam modeling computations are done only once for each beamline configuration upstream of the source plane, and the results are coupled to the patient dosimetry computations for each irradiation via a computed boundary condition specified for the source plane and validated by appropriate measurements.

The measurements reported here were based on a simplified version of the activation foil protocol that was used for earlier INEEL measurements at the FiR 1 epithermal-neutron facility operated by the Technical Research Centre of Finland. ${ }^{9}$ That protocol, in turn, was based on neutron activation analysis techniques ${ }^{10}$ that have been adapted for BNCT applications by the INEEL through extensive experience with several other epithermal-neutron facilities in the U.S. and in Europe. Neutronically thick circular activation foils of various types were used for the measurements. The foil specifications and corresponding neutron activation interactions of interest are listed in Table 1.

Table 1. Activation interactions and foils used for the INEEL/WSU epithermal-neutron beam measurements.

\begin{tabular}{|l|l|l|}
\hline $\begin{array}{c}\text { Neutron } \\
\text { Interaction }\end{array}$ & \multicolumn{1}{|c|}{$\begin{array}{c}\text { Energy Range of } \\
\text { Primary Response }\end{array}$} & $\begin{array}{c}\text { Activation Gamma Energy } \\
\text { of Interest }(\mathrm{keV})\end{array}$ \\
\hline${ }^{115} \mathrm{In}(\mathrm{n}, \gamma)$ & $1 \mathrm{eV}$ Resonance & 1293,1097, and 416 \\
\hline${ }^{197} \mathrm{Au}(\mathrm{n}, \gamma)$ & $5 \mathrm{eV}$ Resonance & 411 \\
\hline${ }^{186} \mathrm{~W}(\mathrm{n}, \gamma)$ & $18 \mathrm{eV}$ Resonance & 686 \\
\hline${ }^{55} \mathrm{Mn}(\mathrm{n}, \gamma)$ & $340 \mathrm{eV}$ Resonance & 847 \\
\hline${ }^{63} \mathrm{Cu}(\mathrm{n}, \gamma)$ & $1 \mathrm{keV}$ Resonance & 511 (Positron) \\
\hline${ }^{45} \mathrm{Sc}(\mathrm{n}, \gamma)$ & $4.5 \mathrm{keV}$ Resonance & 1120,889 \\
\hline${ }^{115} \mathrm{In}\left(\mathrm{n}, \mathrm{n}^{\prime}\right)$ & $430 \mathrm{keV}$ Threshold & 336 \\
\hline
\end{tabular}




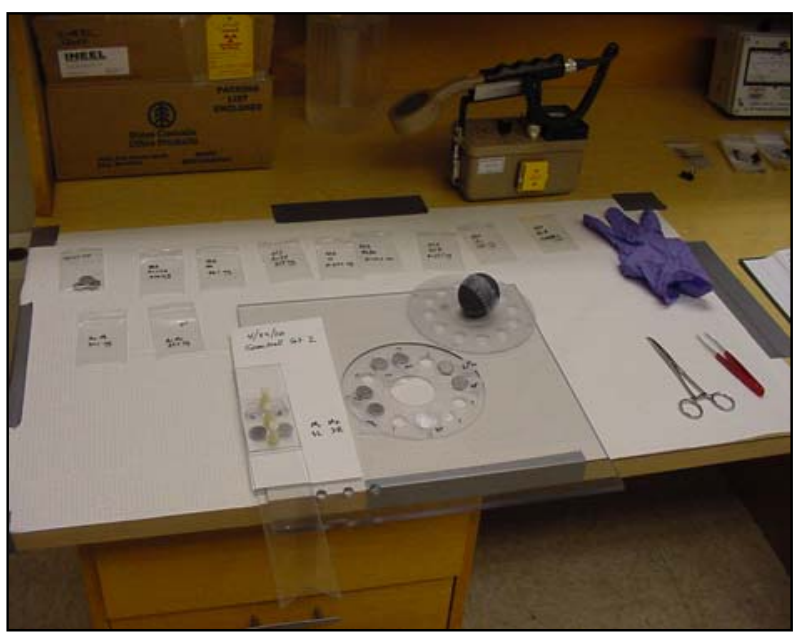

(a)

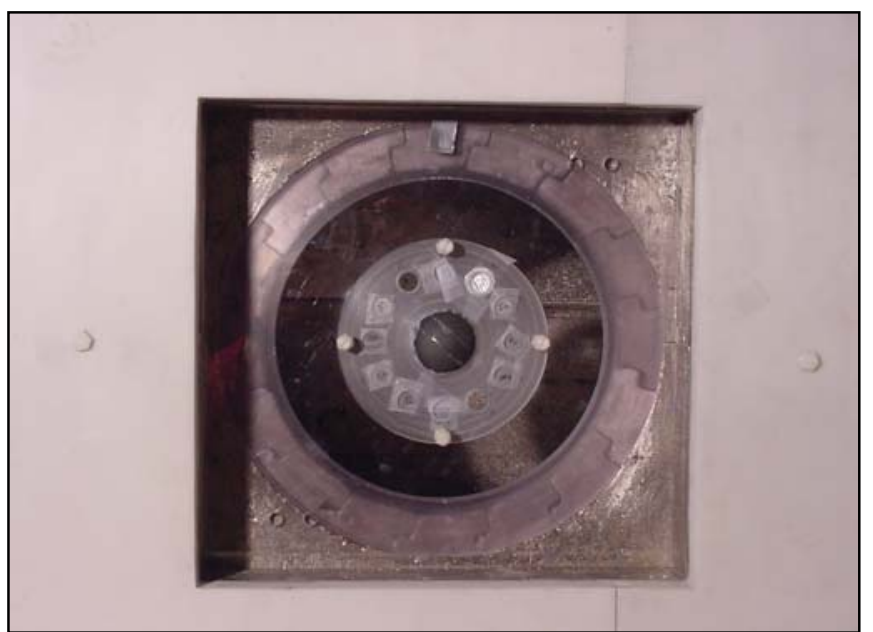

(b)

Figure 6. Source plane activation foil plate (a) positioned in the WSU epithermal neutron beam source plane (b).

Standard 12.7-mm (0.5 in.) diameter $\mathrm{In}, \mathrm{Au}, \mathrm{W}, \mathrm{Mn}, \mathrm{Cu}$, and $\mathrm{Sc}$ foils were used with a Lexan foil positioning plate, as shown in Figure 6. A cadmium cover was placed around one foil of each type to suppress the thermal-neutron response. Thus, each foil responds largely to neutrons having energies at or near the energy of the respective primary resonance of the foil material, as shown in the first six lines of Table 1. The covered foils were placed in outer foil positioning plate locations. An uncovered gold foil was also placed in an outer position of the foil plate to measure the thermal flux. The foils had nominal thicknesses in the range of $0.0254 \mathrm{~mm}$ (0.001 in.) to $0.127 \mathrm{~mm}$ (0.005 in.), depending on the material type.

An additional foil package was used to provide spectral information in the high-energy range. A heavy ( $\sim 5 \mathrm{~g}) 25.4 \mathrm{~mm}(1 \mathrm{in}$.) diameter indium foil was placed in a small hollow boron sphere shown (disassembled) in Figure 7. The composition of the sphere is approximately $93 \%{ }^{10} \mathrm{~B}$ and $7 \%{ }^{11} \mathrm{~B}$ by weight, with a total boron density of $2.6 \mathrm{~g} / \mathrm{cm}^{3}$. This arrangement provides essentially total suppression of thermal and epithermal flux within the inner cavity of the boron sphere. Thus, an artificial threshold above the resonance energy range is imposed on the neutron capture reactions in the foil within the sphere. Since the activation gamma emissions that arise from neutron capture in indium are suppressed, the relatively weak $336 \mathrm{keV}$ gamma line from inelastic scatter in indium, which is of crucial interest in these measurements, is much more prominent in the spectrum of the activated indium foil. The activated indium foil is also much easier to handle when the boron sphere is used in this manner, since the induced activity is significantly lower than it would be without the sphere. The inside diameter of the boron sphere is approximately $2.8 \mathrm{~cm}(1.1 \mathrm{in}$.). The outside diameter is approximately $4.75 \mathrm{~cm}(1.9 \mathrm{in}$.). The boron sphere assembly was placed in the center of the foil positioning plate, as shown in Figure $6 \mathrm{~b}$. 


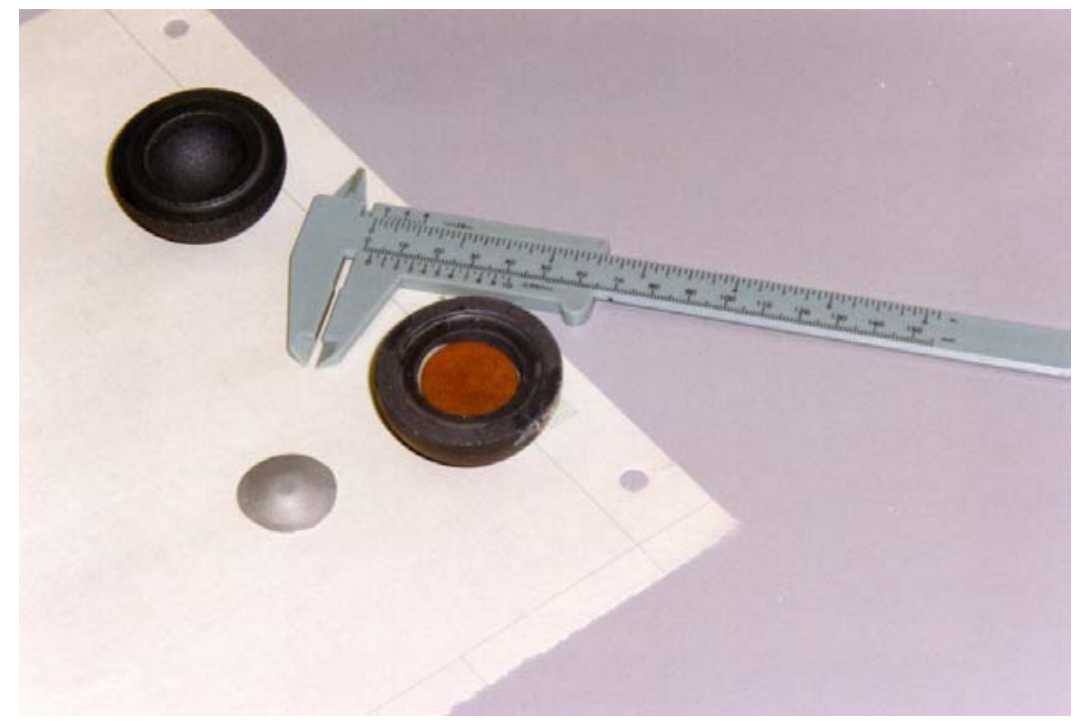

Figure 7. Boron sphere used to suppress low-energy neutron flux.

Use of the foils as described provided 8 basic neutron response functions having a useful degree of linear independence. These response functions were:

1. Resonance capture in the $\mathrm{Sc}, \mathrm{Cu}, \mathrm{Mn}, \mathrm{W}, \mathrm{Au}$ and In foils, all with thermal neutron capture suppressed by cadmium ( 6 responses);

2. Total neutron capture in the single uncovered gold foil (1 response);

3. Inelastic scatter in the indium foil in the boron sphere (1 response).

The measurements reported here are based on the results of a one-hour irradiation at a reactor power of $1 \mathrm{MW}$.

The irradiated foils were assayed at WSU using a standard high-purity germanium (HPGe) gamma spectrometry system (Canberra/Genie). The induced activities in the free-beam foils were computed from the photopeak areas and system efficiencies based on calibration of the spectrometer using a National Institute Standards and Technology (NIST)-traceable mixed europium-antimony calibration source. The measured activity of the heavy indium foil in the boron sphere was corrected for gamma self-shielding using an escape fraction at $336 \mathrm{keV}$, calculated using a combination of MCNP computations and handbook data for the specific source-detector geometry that was used for the assay. This factor was $0.90 \pm 0.01$. The activity of the indium foil in the cadmium cover was taken as the average of the results derived separately from the measured activities of the three primary gamma lines. The measured activation rates of the various foils were then used to estimate the neutron spectrum by way of a direct least-squares matrix unfolding procedure, documented in Reference 11.

Superheated Nucleation Detector Measurements in the Source Plane. The free-beam measurements outlined above were based on standard activation techniques. This approach is simple and capable of very high accuracy in a piecewise integral sense. However, it has a significant disadvantage when additional detail is desired for neutrons in the energy range of 
$10 \mathrm{keV}$ to $400 \mathrm{keV}$. Convenient materials with suitable characteristic activation responses (either resonance or threshold) are not readily available for detailed measurements in this energy range. Other methods, such as proton-recoil chambers, time-of-flight measurements, etc., are available for obtaining detail in this energy range, but these also have their characteristic disadvantages. However, one promising low-cost alternate technique that appears to offer a solution, and for which relatively new implementing technologies have recently become available, is based on counting of radiation-induced nucleation sites in specialized superheated materials. ${ }^{12,13}$ Some exploratory measurements using this technique were completed at WSU during the year.

The particular approach used for the measurements presented here features the use of a RemSpec $^{\mathrm{TM}}$ Superheated Drop Detector (SDD) spectrometer available from Apfel Enterprises, Inc. There are SDD dosimeter materials available for this device that are totally photoninsensitive. These materials have thresholds for neutron detection between $50 \mathrm{keV}$ and $1 \mathrm{MeV}$ in the case of one medium, and $500 \mathrm{keV}$ to $10 \mathrm{MeV}$ in the case of a second medium. In each case, the threshold energy can be fine-tuned by varying the temperature of the medium, and the response function of the medium above the threshold can be quantified. The RemSpec ${ }^{\mathrm{TM}}$ incorporates an SDD-containing vial, a temperature control system, and an acoustic sensor that records the formation of each nucleation site in real-time, along with appropriate control and data acquisition software operating under the Microsoft Windows ${ }^{\mathrm{TM}}$ environment. Since the threshold and the response function of the SDD media are known at each temperature, it is possible to unfold the bubble formation data to produce a measured neutron spectrum within the energy range over which the medium is sensitive. This offers an interesting alternative to activation spectrometry that should have utility for some radiobiological studies at WSU. The SDD devices can provide data in the crucial low-energy range of the neutron spectrum, just above the upper epithermal cutoff. This energy range is of current radiobiological interest because of the relative lack of validated biological effectiveness data. ${ }^{14}$

Figure 8 shows the RemSpec ${ }^{\mathrm{TM}}$ detector assembly positioned in the source plane of the WSU beam. The actual detector material is enclosed in a tube that is incorporated into an insulated heating unit to vary the temperature, as discussed above. An ion chamber (RadCal Corporation) is also suspended in the beam to provide an indication of the gamma dose rate in the free beam. The reactor power for the RemSpec ${ }^{\mathrm{TM}}$ irradiations was on the order of a few tens of watts, in order to maintain a count rate within the specifications of the instrument. 


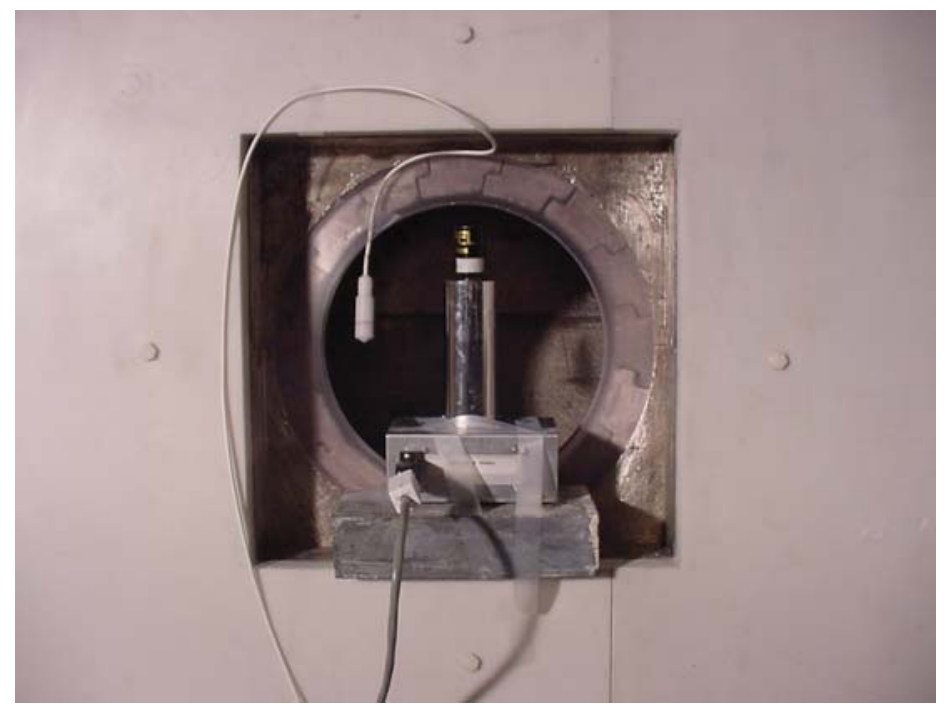

Figure 8. RemSpec ${ }^{\mathrm{TM}}$ superheated nucleation spectrometer unit positioned in the source plane at WSU.

Activation Foil Measurements in a Shaped Exit Aperture. Additional free beam measurements were completed for the case of a $10.16 \mathrm{~cm}$ diameter circular aperture produced by installation of suitable beam shaping plates in the bismuth collar. Two sets of beam aperture plates designed to fit in the bismuth collar are shown in Figure 9, along with three Lucite phantoms used for various beam performance measurements. The aperture plates provide various circular beam apertures, with the diameter depending on how many plates are installed. The pink colored plates are composed of borated polyethylene ( $5 \%$ natural boron by weight), while the white plates are composed of enriched Lithium-6 polyethylene $\left({ }^{6} \mathrm{Li}\right.$ density of $\left.50 \mathrm{mg} / \mathrm{cm}^{3}\right)$. In the case of the measurements presented here, the lithiated polyethylene plates were used. These are shown in Figure 10, installed in the bismuth collar piece.

The various foils listed in the first six lines of Table 1 were positioned in the exit plane aperture using a device designed by the INEEL for this type of measurement and machined from an aluminum disk. This device, referred to as a "foil wheel", is shown in Figure 11. The six cadmium-covered packages were placed in the six outer foil wheel positions. An uncovered gold foil was placed in the center position of the foil wheel, again to measure the thermal flux. Reproducible positioning of the foil wheel in the plane of the beam exit port was accomplished using a simple mounting plate placed in the beam exit port, as shown in Figure 12. The boron sphere, again with a heavy indium foil inside, was positioned in the exit port using a similar plate, as shown in Figure 13. The foil wheel and boron sphere were irradiated in two separate irradiations at a reactor power of $1 \mathrm{MW}$. 


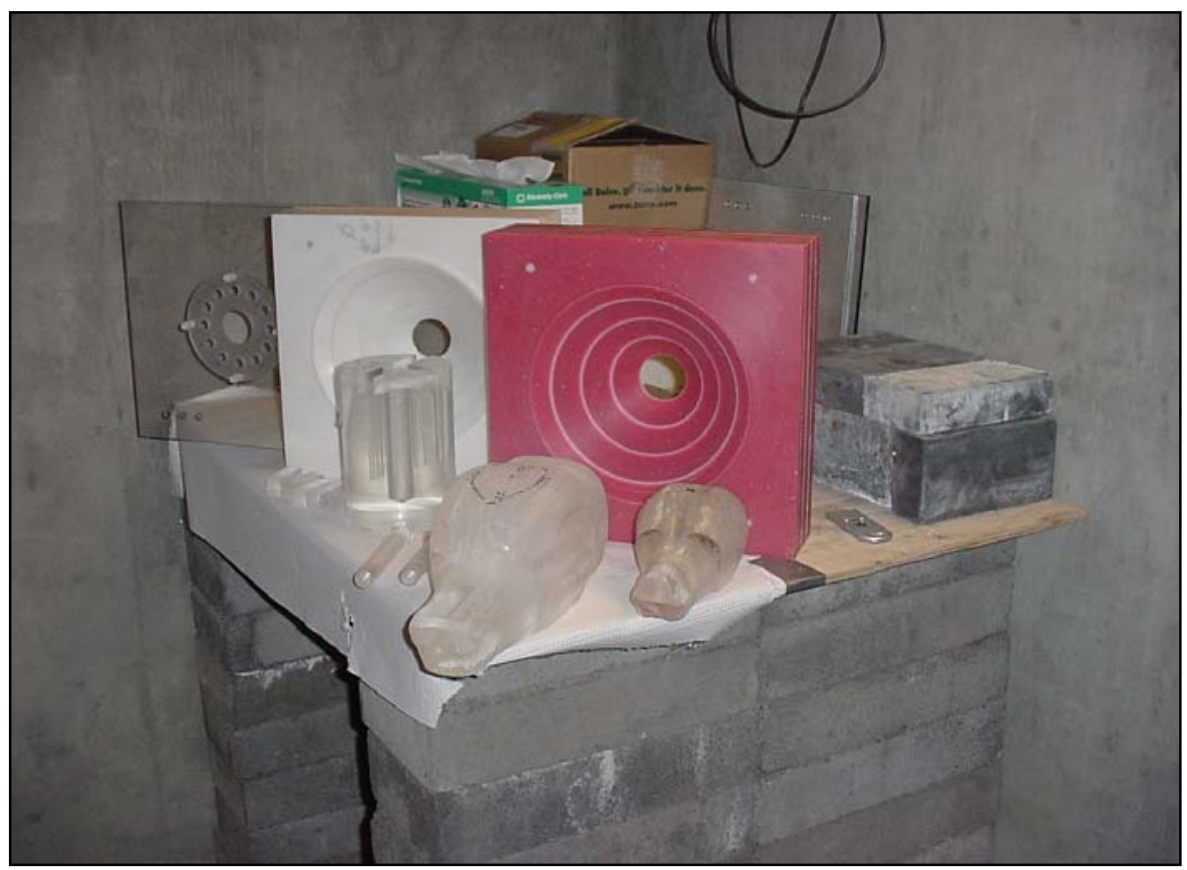

Figure 9. Beam aperture plates and various Lucite phantoms used at WSU.

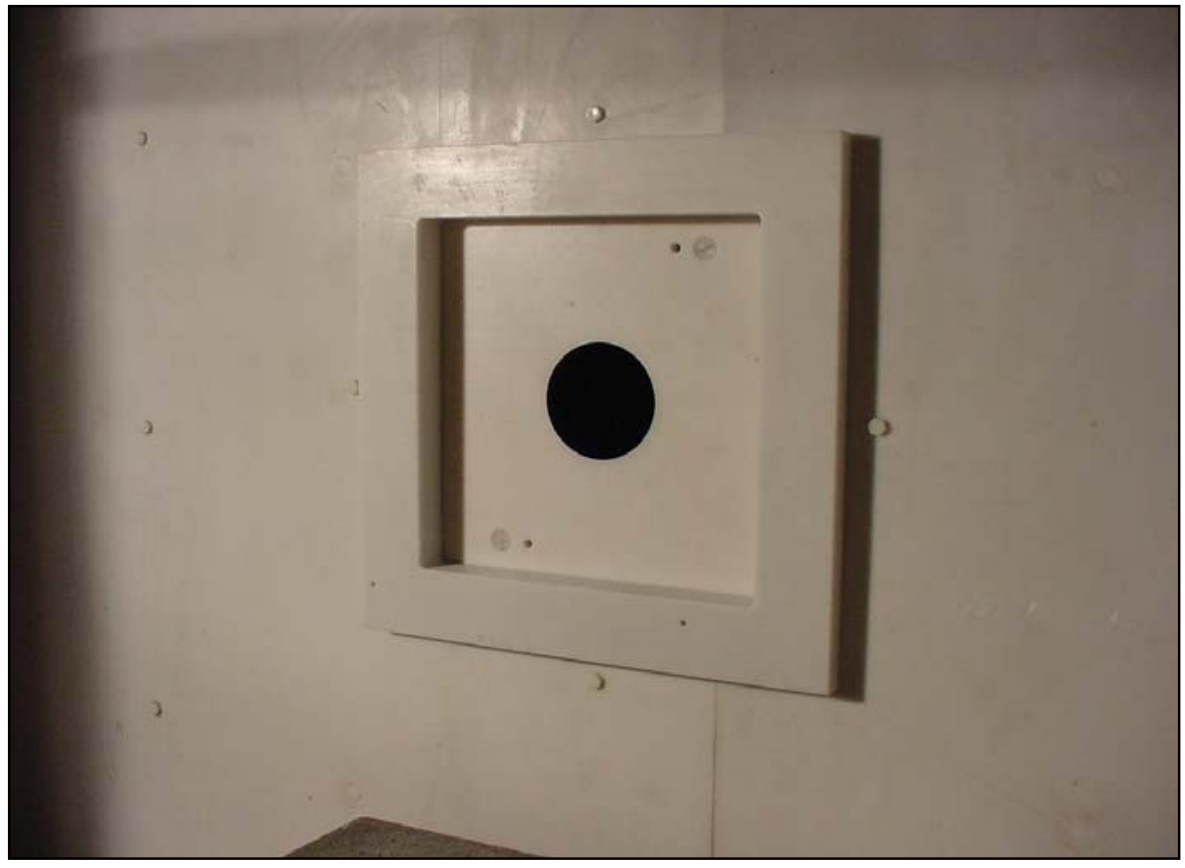

Figure 10. Lithiated polyethylene beam aperture plate arrangement for the $10.16 \mathrm{~cm}$ diameter beam exit aperture at WSU. 


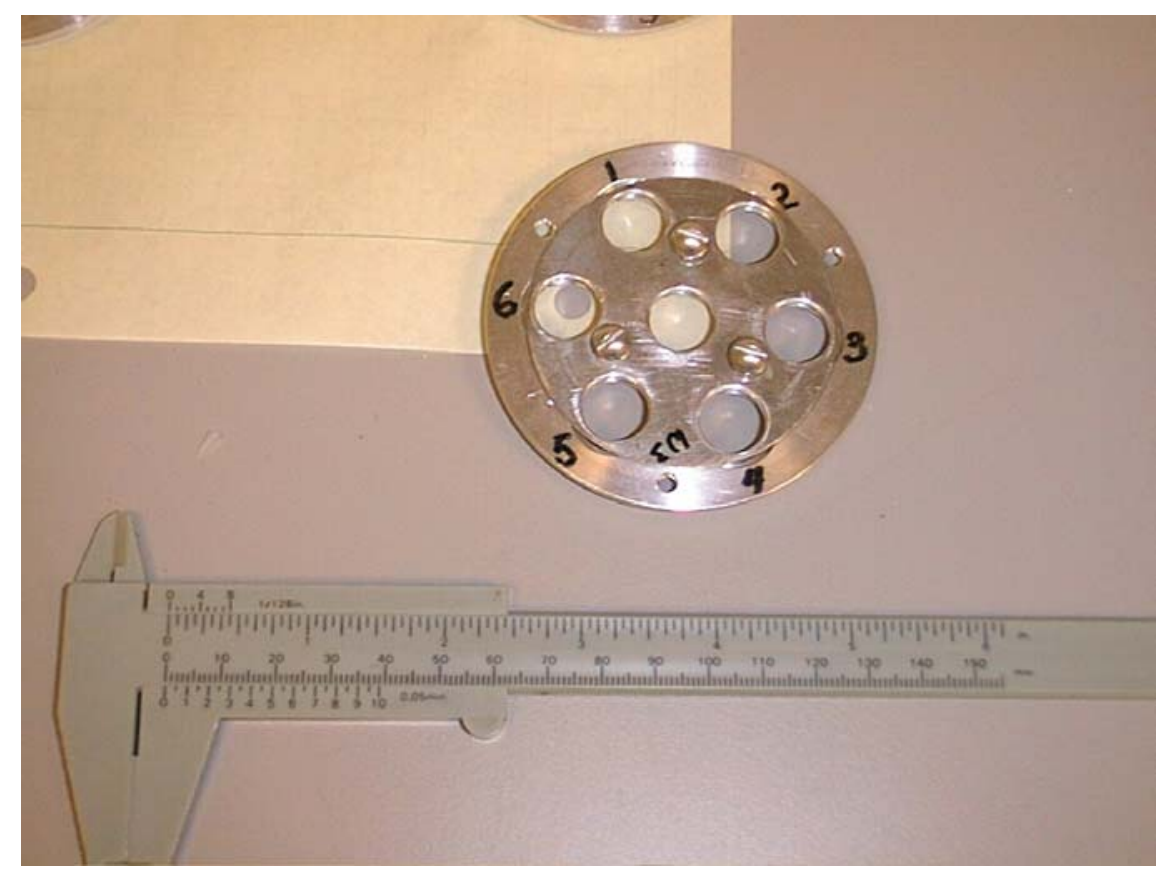

Figure 11. Foil positioning device (foil wheel)for exit aperture spectral measurements at WSU.

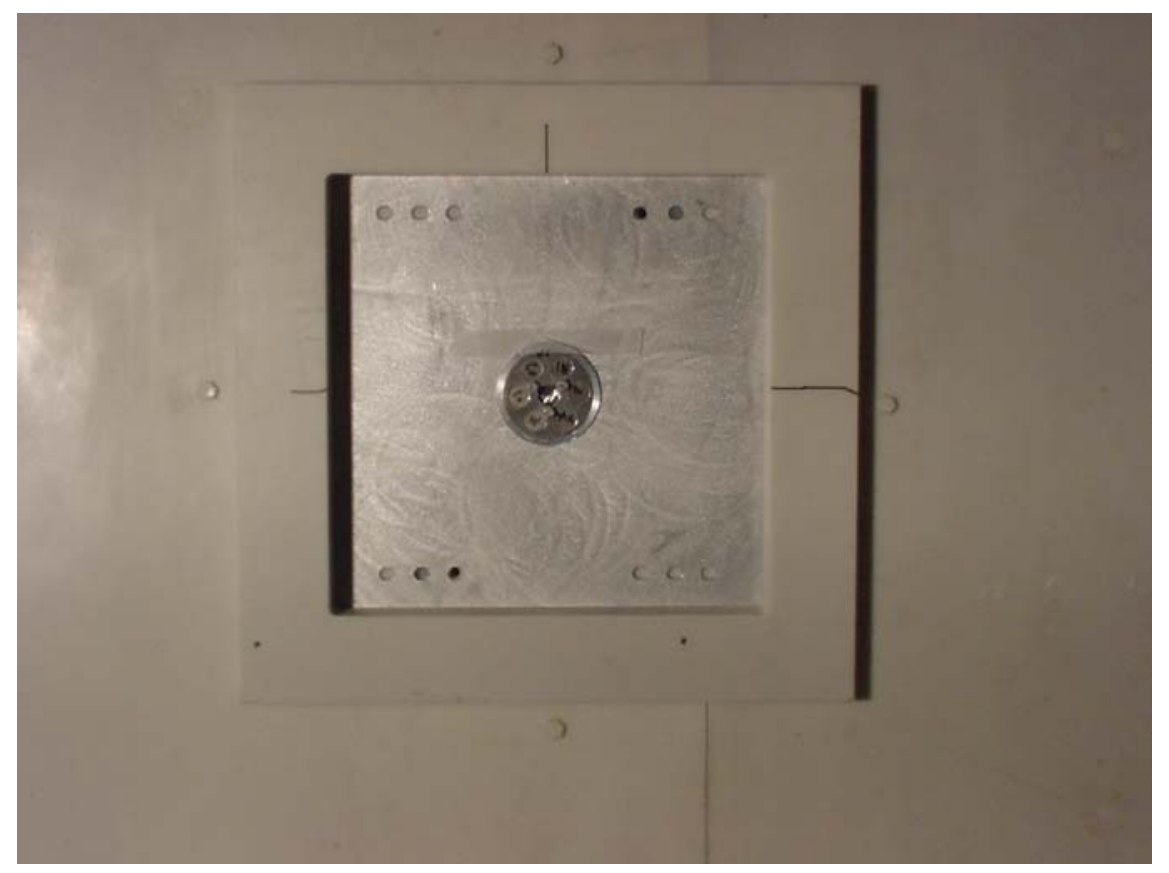

Figure 12. Foil wheel in the $10.16 \mathrm{~cm}$ beam exit aperture at WSU. 


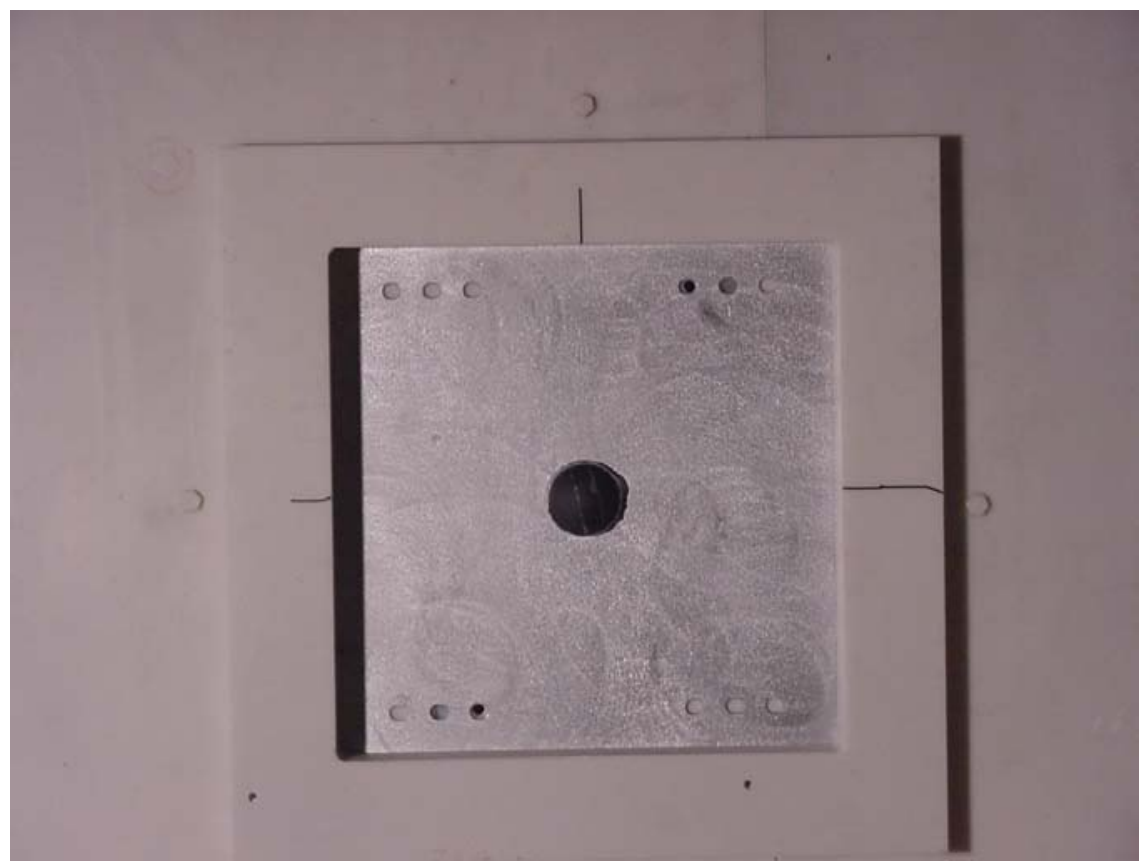

Figure 13. Boron sphere positioned in the $10.16 \mathrm{~cm}$ beam exit aperture at WSU.

Phantom Measurements. Measurements of the neutron flux-depth distribution produced by the WSU beam in a standard cylindrical Lucite phantom were also performed using activation techniques. Figure 14 shows the phantom, a lucite cylinder of diameter $12.7 \mathrm{~cm}$ and length 18.2 $\mathrm{cm}$. The phantom has a removable central insert with holes drilled at various axial intervals for placement of flux wires. The phantom was positioned adjacent to the WSU $10.16 \mathrm{~cm}$ exit port, as shown in Figure 15. The flux wires used for the measurements reported here were approximately $10 \mathrm{~mm}$ in length and $1 \mathrm{~mm}$ in diameter. Each wire had a mass of approximately $73 \mathrm{mg}$ and was composed of copper alloyed with 1.55 percent gold by weight. This provided two linearly-independent activation responses (neutron absorption in ${ }^{63} \mathrm{Cu}$ and ${ }^{197} \mathrm{Au}$ ) that were used to unfold a two-group (thermal and above-thermal) neutron spectrum at each flux wire location. Flux wires were placed at axial depths of $0.0 \mathrm{~cm}$, and at subsequent $1-\mathrm{cm}$ intervals along the beam centerline, starting at a depth of $1.5 \mathrm{~cm}$. A two-group spectrum was unfolded at each axial depth using the same method as was used for the multigroup free-beam spectrum unfolding computations. The required unfolding parameters for the flux wire materials were computed at each axial location using an MCNP model of the phantom with a flux boundary condition representing the WSU neutron source incident on the end. 


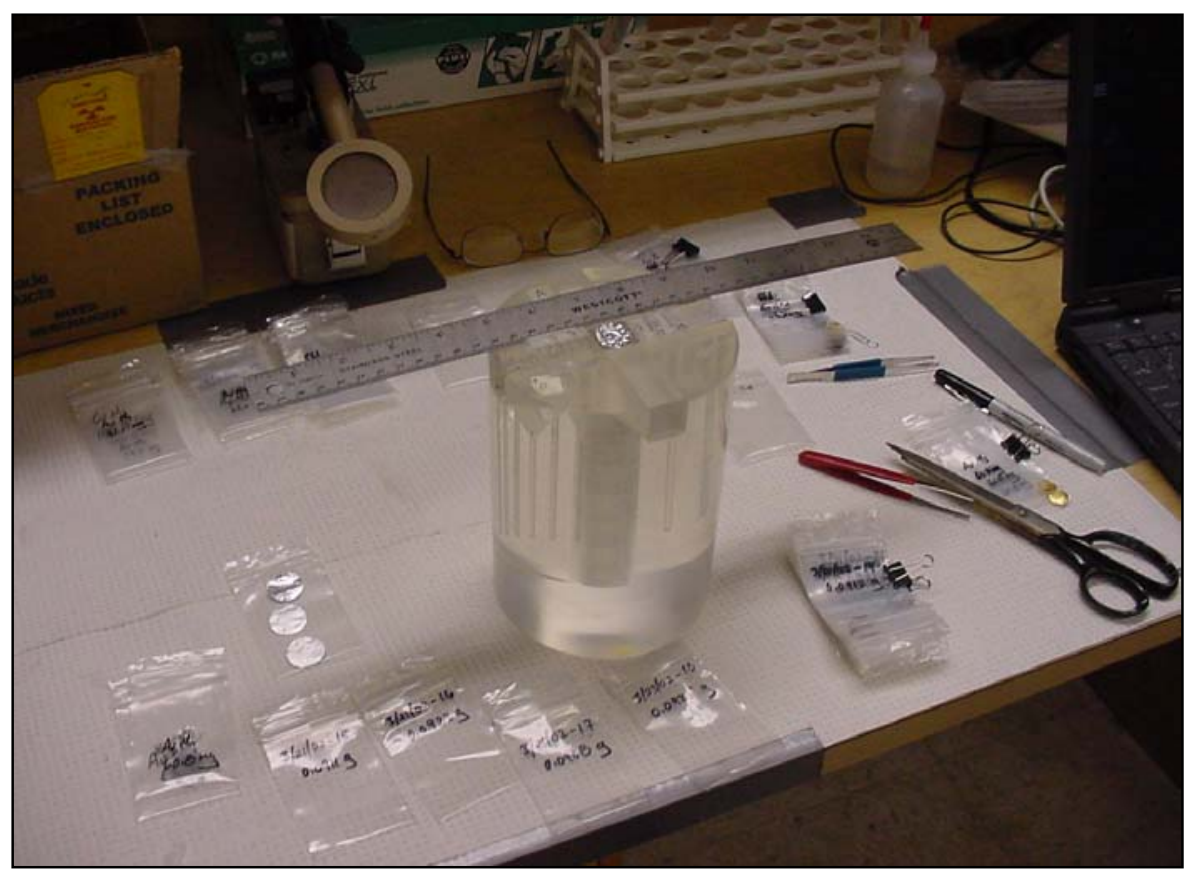

Figure 14. INEEL Lucite phantom.

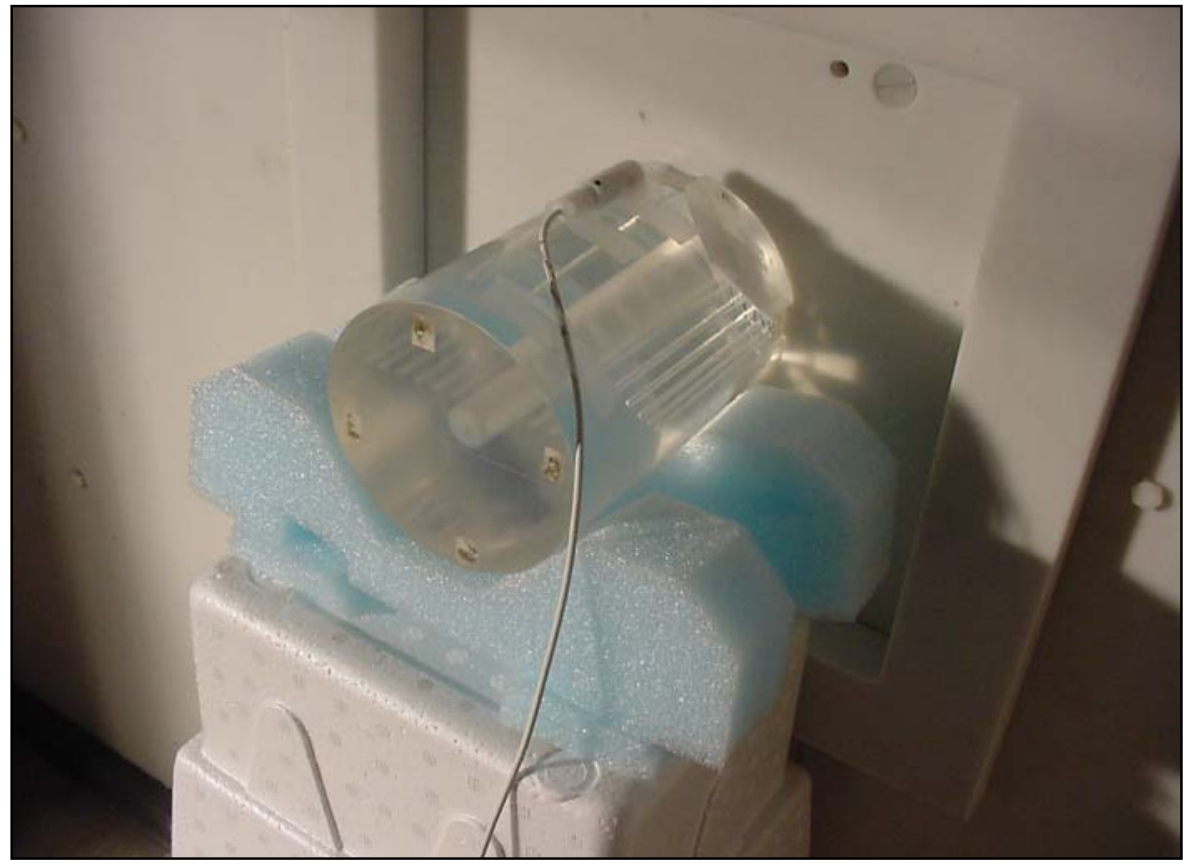

Figure 15. Positioning of the Lucite phantom in the WSU beam exit aperture. 


\section{RESULTS}

Free-Beam Spectra in the Source Plane and the $10.16 \mathrm{~cm}$ Exit Plane. The measured saturation activities of the various foils used for the two free-beam spectral measurements are shown in Table 2. The statistical uncertainties given in this table include the uncertainty of the spectrometer calibration as well as the uncertainty of the activity measurement for each foil. These were used to derive the spectrum of the neutron flux at the measurement location using the previously-referenced direct few-group matrix unfolding procedure. In this procedure, the $a$ priori flux was computed using a DORT ${ }^{8}$ model of the beamline, with cross section data taken from the BUGLE- $80^{15}$ library.

Table 2. Foil saturation activities measured at a WSU reactor power of $1 \mathrm{MW}$.

\begin{tabular}{|c|c|c|c|}
\hline $\begin{array}{c}\text { Neutron } \\
\text { Interaction }\end{array}$ & $\begin{array}{c}\text { Saturation Activity } \\
\text { (Bq/atom) Measured in } \\
\text { the Source Plane }\end{array}$ & $\begin{array}{c}\text { Saturation Activity } \\
\text { (Bq/atom) Measured in } \\
\text { the Exit Aperture }\end{array}$ & $\begin{array}{c}\text { Ratio of Exit Plane } \\
\text { Activation Rate to Source } \\
\text { Plane Activation Rate }\end{array}$ \\
\hline${ }^{197} \mathrm{Au}(\mathrm{n}, \gamma)^{1}$ & $4.29 \times 10^{-14}(2.1 \%)$ & $3.38 \times 10^{-14}(2.1 \%)$ & 0.79 \\
\hline${ }^{115} \mathrm{In}(\mathrm{n}, \gamma)^{2}$ & $5.32 \times 10^{-14}(2.2 \%)$ & $4.34 \times 10^{-14}(2.1 \%)$ & 0.82 \\
\hline${ }^{197} \mathrm{Au}(\mathrm{n}, \gamma)^{2}$ & $3.89 \times 10^{-14}(2.1 \%)$ & $3.06 \times 10^{-14}(2.1 \%)$ & 0.79 \\
\hline${ }^{86} \mathrm{~W}(\mathrm{n}, \gamma)^{2}$ & $2.13 \times 10^{-14}(2.2 \%)$ & $1.78 \times 10^{-14}(2.9 \%)$ & 0.84 \\
\hline${ }^{55} \mathrm{Mn}(\mathrm{n}, \gamma)^{2}$ & $6.02 \times 10^{-16}(2.2 \%)$ & $4.36 \times 10^{-16}(2.2 \%)$ & 0.72 \\
\hline${ }^{63} \mathrm{Cu}(\mathrm{n}, \gamma)^{2}$ & $1.64 \times 10^{-16}(3.2 \%)$ & $1.22 \times 10^{-16}(3.3 \%)$ & 0.74 \\
\hline${ }^{45} \mathrm{Sc}(\mathrm{n}, \gamma)^{2}$ & $5.79 \times 10^{-16}(5.0 \%)$ & $4.19 \times 10^{-16}(6.5 \%)$ & 0.72 \\
\hline${ }^{115} \mathrm{In}\left(\mathrm{n}, \mathrm{n}^{\prime}\right)^{3}$ & $2.49 \times 10^{-19}(2.8 \%)$ & $2.31 \times 10^{-19}(3.5 \%)$ & 0.93 \\
\hline
\end{tabular}

1 Bare foil.

2 Cadmium covered foil.

3 Foil in boron sphere.

4 Percentage uncertainty $(1 \sigma)$ given in parentheses.

Figure 16 shows an unfolded 7-group free-field unperturbed neutron spectrum in the source plane, projected by the unfolding process to the center of the foil positioning plate. The computed 47-group a-priori neutron spectrum, normalized to $1 \mathrm{MW}$, is also shown for comparison. The broad group structure used for the unfolded spectrum presented in Figure 16 was selected to provide a well-conditioned unfolding matrix, and to permit an accurate integration of the measured spectrum in the epithermal energy range to determine the total epithermal neutron flux. 


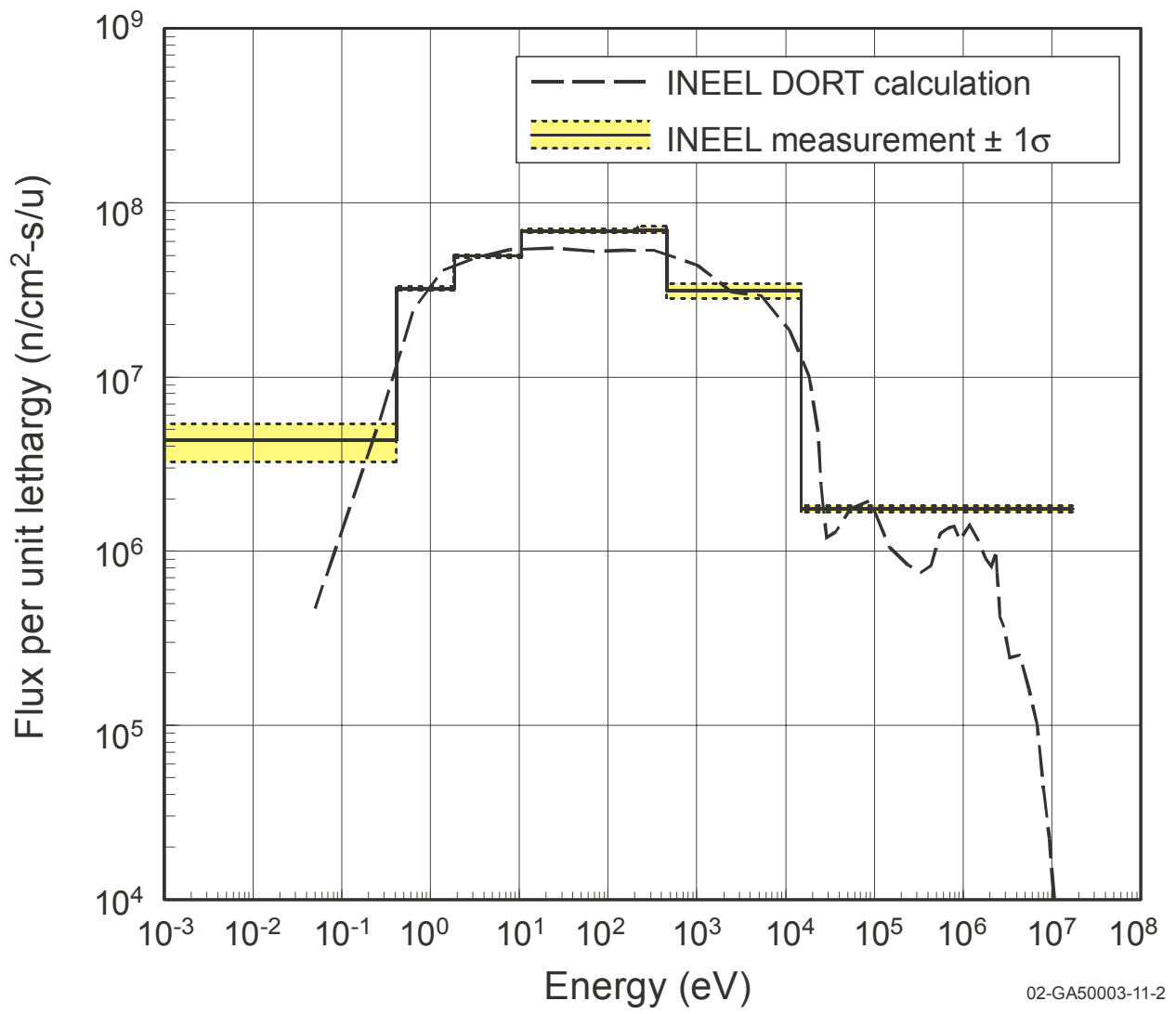

Figure 16. Unfolded free beam neutron spectrum in the source plane obtained by direct fitting for the WSU epithermal neutron beam facility. The reactor power was $1 \mathrm{MW}$.

It will be observed that the propagated uncertainty associated with the thermal flux $(\mathrm{E}<0.414 \mathrm{eV})$ in Figure 16 is quite large. This is a result of the fact that the thermal flux is very small compared to the remainder of the spectrum. The measured activities of the gold foils with and without $\mathrm{Cd}$ covers differ by only a few percent. This leads to a magnification of the uncertainty when the thermal flux is estimated from these foil activities in the spectral unfolding process.

Integrating the measured curve shown in Figure 16 between the standard epithermal energy limits of $0.414 \mathrm{eV}$ and $10 \mathrm{keV}$ produces a measured epithermal neutron flux of $4.94 \times 10^{8} \mathrm{n} / \mathrm{cm}^{2}$ sec at $1 \mathrm{MW}$ with a propagated uncertainty of approximately $5 \%(1 \sigma)$. This is well within the expected range based on the design computations. It may be noted that this flux is expected to increase by a factor of between 2 and 3 when the WSU reactor fuel element loading pattern is reconfigured for optimum beam performance.

A simple, but widely used, metric for the biophysical quality of reactor-based epithermal-neutron beams is based on dividing the free-field neutron KERMA rate of the beam (integrated over all energies above thermal) by the useful epithermal-neutron flux, measured in the free field. This parameter is an indicator of the non-selective neutron background dose that will be produced in tissue by the above-thermal spectral component of the beam itself, in the absence of any neutron capture agent that may be administered to the patient. It is computed here for WSU using the measured flux spectrum in conjunction with broad group KERMA factors based on data for the 
components of standard tissue available on File 27 of the BUGLE ${ }^{15}$ cross section library. Finegroup neutron KERMA factors are averaged over the broad unfolding group structure, using the a-priori spectrum as a weighting function, to produce the necessary information. This procedure yields a measured free-beam, spectral-averaged, total neutron KERMA factor of $2.84 \times 10^{-11} \mathrm{cGy}$ per unit useful epithermal-neutron flux, with an estimated uncertainty of about $7 \%$. This is in excellent agreement with the computed value of $2.75 \times 10^{-11} \mathrm{cGy}-\mathrm{cm}^{2}$. Approximately $91 \%$ of the background neutron dose results from proton recoil. The remainder is from other recoil components. Additional background dose in tissue is produced by non-selective thermal-neutron induced components, primarily from neutron capture in nitrogen and hydrogen, but these components are not directly indicative of the intrinsic biophysical quality of the incident beam. They must be taken into account in treatment planning, but they are not normally included in the free-beam quality parameter discussed here.

Figure 17 shows the unfolded spectrum in the $10.16 \mathrm{~cm}$ exit plane. The shape of the spectrum is similar, as expected, but the estimated flux integral in the epithermal range is now $3.84 \times 10^{8}$ $\mathrm{n} / \mathrm{cm}^{2}$-sec at $1 \mathrm{MW}$, about $77 \%$ of the integrated flux in the source plane. This intensity loss is primarily the result of beam divergence over a distance corresponding to the total thickness of the aperture plates used.

Superheated Nucleation Detector Measurements. Figure 18 shows results from the RemSpec ${ }^{\mathrm{TM}}$ measurements. In this case, the fluxes are plotted on an expanded scale to show detail above about $30 \mathrm{keV}$, the lower threshold of the energy range that is detectable by the instrument. To obtain the results shown in this figure, the RemSpec ${ }^{\mathrm{TM}}$ detector response curves from the user manual were digitized to an 84-group, equal-lethargy, structure covering the energy range from $30 \mathrm{keV}$ to $10 \mathrm{MeV}$. The a-priori flux from the baseline beam design computations was likewise remapped into this group structure. The spectrum implied by the RemSpec ${ }^{\mathrm{TM}}$ responses was estimated using a variation of the Sand-II empirical adjustment method, ${ }^{16}$ as described by Draper. ${ }^{17}$ The measured results were normalized such that the integral of the flux above $630 \mathrm{keV}$ was set equal to the corresponding estimated value from the free beam foil measurements. This procedure was used for consistency because $630 \mathrm{keV}$ is the energy threshold for the SDD response curve that most closely matched that of the indium inelastic scatter interaction used to independently measure the high-energy part of the flux spectrum, as described previously. 


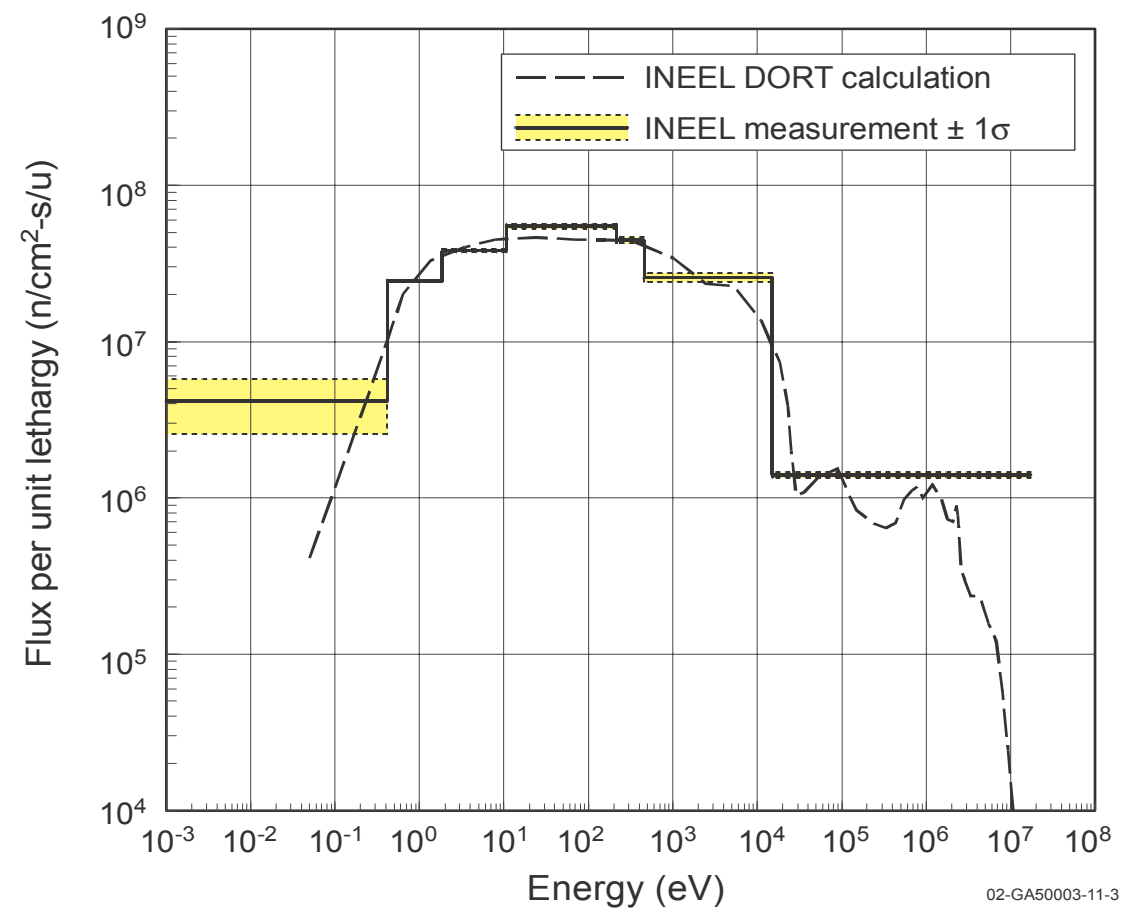

Figure 17. Unfolded free beam neutron spectrum in the $10.16 \mathrm{~cm}$ diameter beam aperture obtained by direct fitting for the WSU epithermal neutron beam facility. The reactor power was $1 \mathrm{MW}$.

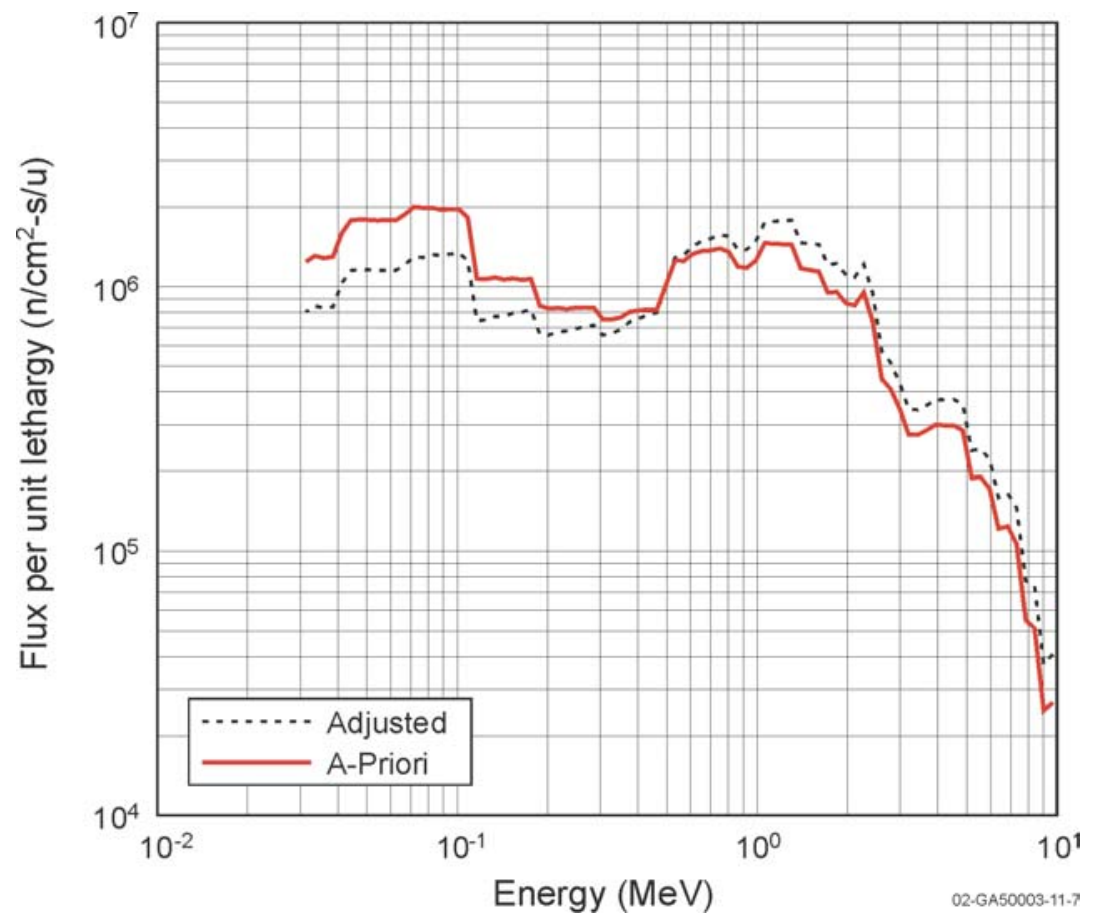

Figure 18. Results of the RemSpec ${ }^{\mathrm{TM}}$ measurements for the WSU epithermal neutron beam. 
The adjustment procedure produces a somewhat harder measured spectrum compared to the $a$ priori curve, as seen in Figure 18. Neutrons are shifted to the energy range above about $500 \mathrm{keV}$ in order to produce a better fit to the observed detector responses. This appears reasonable based on experience with several reactor-based epithermal beams over the years. It is also consistent with results reported by d'Ericco, et al., ${ }^{18}$ for a measurement of the epithermal beam at the Studsvik laboratory in Sweden using the same basic experimental approach.

Phantom Measurements. Figure 19 shows the unfolded thermal and above-thermal fluxes produced in the cylindrical phantom. Agreement with the a-priori computations is quite good at most points. Furthermore, the ratio of the peak thermal neutron flux generated in the phantom relative to the magnitude of the incident epithermal flux is consistent with expectations.

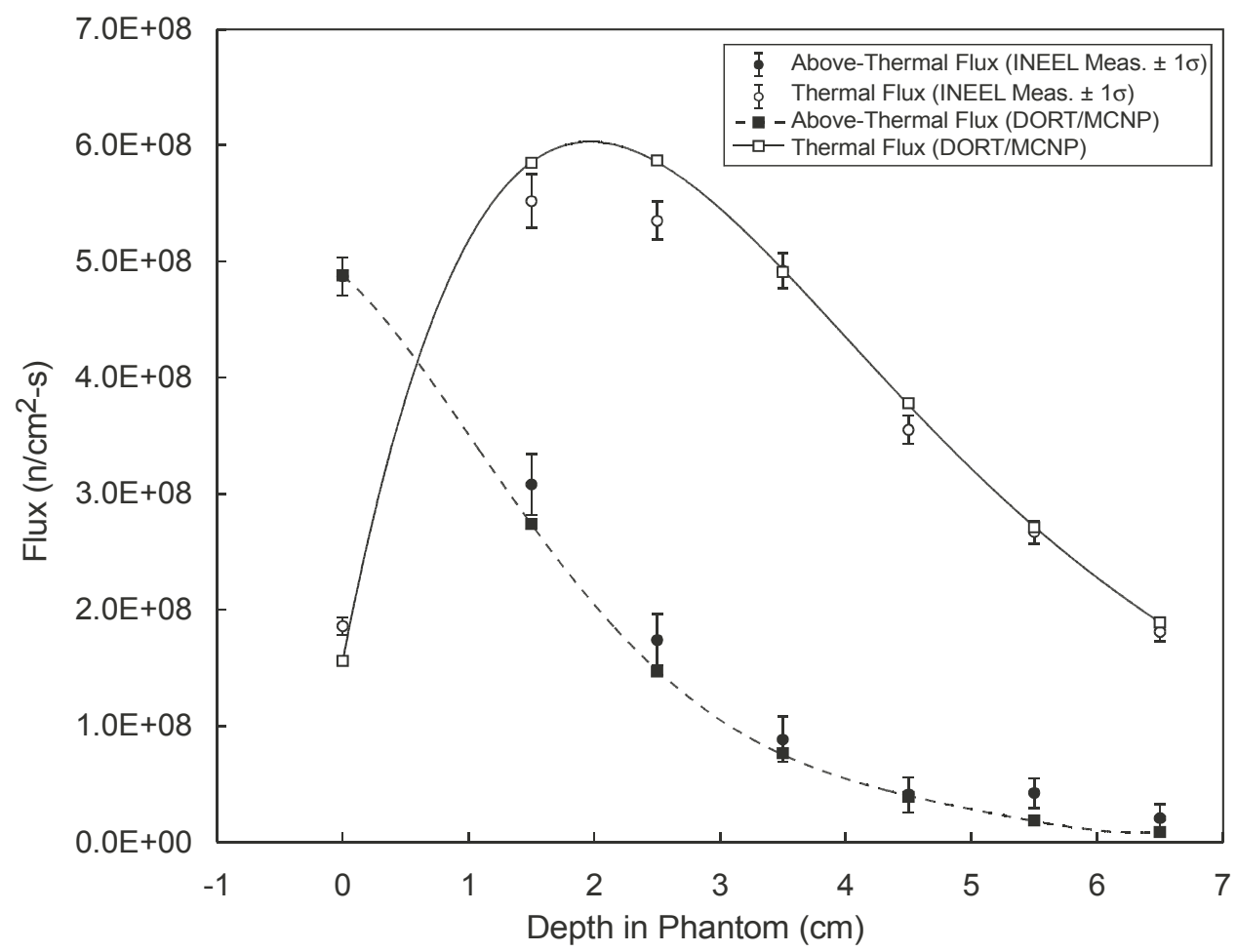

Figure 19. Fluxes in the INEEL cylindrical Lucite phantom irradiated in the $10.16 \mathrm{~cm}$ aperture of the WSU epithermal neutron beam facility. The reactor power was $1 \mathrm{MW}$.

\section{DISCUSSION}

The WSU facility is the third clinical-scale reactor-based epithermal-neutron source for BNCT research that has been constructed in the U.S. With the recent decision to decommission the Brookhaven Medical Research Reactor, the new WSU neutron source and a recently constructed epithermal beam facility at the Massachusetts Institute of Technology ${ }^{19}$ are the only two currently operating, reactor based facilities of this type in the U.S. The measurements reported here confirm the expected neutronic performance of this facility. 
Planned activities at WSU for 2003 include, 1) Completion of an independent experimental evaluation of the WSU epithermal beam performance in collaboration with the European team of the International BNCT Dosimetry Exchange and, 2) Installation of neutron thermalization components in the beamline and initial testing of the resulting thermal neutron source configuration, which is intended for use with small-animal studies. In anticipation of the Dosimetry Exchange activity, a Scanditronix clinical water phantom with a 3-dimensional ion chamber positioning system was installed in late 2002 at WSU, as shown in Figure 20. A set of Far West paired tissue-equivalent and graphite ion chambers (784-TTW and 773-TGW, respectively) was also installed and some initial testing of these was completed. Finally, reloading of the reactor core to produce an increase of the useful flux by about a factor of three at the beam exit port, in either the epithermal or thermal configuration, is currently scheduled for late 2003 or early 2004. Recharacterization of the upgraded beam in both the thermal and epithermal modes will then be completed.
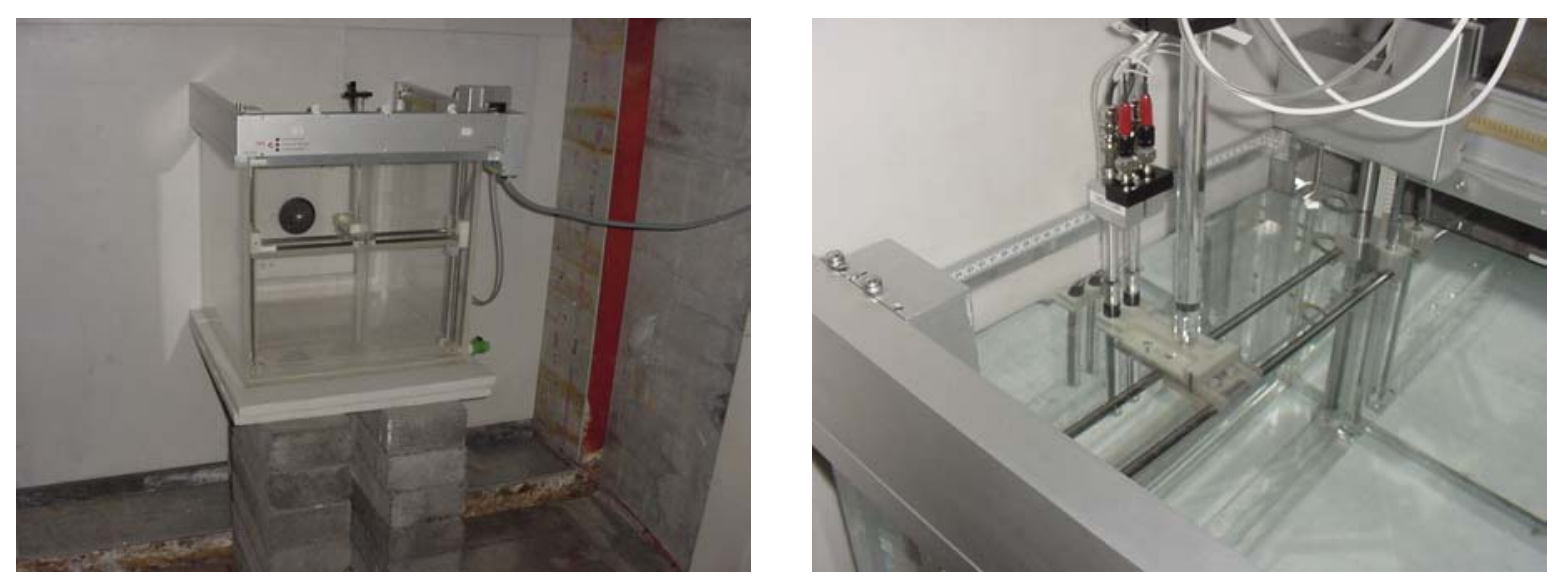

Figure 20. Water phantom at WSU (left), with detail of paired ion chamber arrangement (right).

\section{REFERENCES}

1. P.R. Gavin, S.L. Kraft, C.E. DeHann, C.D. Swartz, M.L. Griebenow, "Large Animal Normal Tissue Tolerance with Boron Neutron Capture," Int. J. Radiat. Oncol. Bio. Phys., 28:10991106, 1994.

2. F.J. Wheeler, D.W. Nigg, "Feasibility Study for an Epithermal-Neutron Beam Facility at the Washington State University Radiation Center," EGG-NRE-11296, Idaho National Engineering Laboratory, 1994.

3. T.D. Burns, Jr., "A Monte Carlo Model System for Core Analysis and Design at the Washington State University Radiation Center," INEL-95/0458, Idaho National Engineering Laboratory, 1996. 
4. J.R. Venhuizen, et al., "Construction Progress of an Epithermal Neutron Beam for Preclinical BNCT Research at Washington State University," In: J.R. Venhuizen (ed.), "INEEL BNCT Research Program Annual Report - 2000," INEEL-EXT-01-00204, March 2001.

5. J.R. Venhuizen, et al., "Construction of an Epithermal Neutron Beam for Preclinical BNCT Research at Washington State University," In: J.R. Venhuizen (ed.), "INEEL BNCT Research Program Annual Report - 2001," INEEL-EXT-02-00060, April 2002.

6. I. Auterinen, P. Hiismäki, "Epithermal BNCT Neutron Beam Design for a TRIGA II Reactor," Advances in Neutron Capture Therapy, Plenum Press, New York, 1993, Pages 8184.

7. J.F. Briesmeister, "MCNP - A General Monte Carlo N-Particle Transport Code, Version 4A," LA-12625-M, Los Alamos National Laboratory, 1993.

8. W.A. Rhoades, et al., "TORT-DORT: Two and Three-Dimensional Discrete-Ordinates Transport," CCC-543 Radiation Shielding Information Center, Oak Ridge National Laboratory, 1993.

9. D.W. Nigg, Y.D. Harker, J.K. Hartwell, C.A. Wemple, T. Seren, I. Auterinen, P. Kotiluoto, P. Hiismaki, T. Sepala, M. Kortesniemi, S Savolainen, R. Risler, "Collaborative Neutronic Performance Characterization of the FiR-1 Clinical Epithermal Neutron Beam Facility for BNCT," In: J.R. Venhuizen (ed.), "INEEL BNCT Research Program Annual Report - 1998," INEEL-EXT-99-00293, April 1999.

10. Y.D. Harker, et al., "Spectral Characterization of the Epithermal Neutron Beam at the Brookhaven Medical Research Reactor," Nuclear Science and Engineering, 110:355-368, 1992.

11. D.W. Nigg, C.A. Wemple, R. Risler, J.K. Hartwell, Y.D. Harker, G.E. Laramore, "Modification of the University of Washington Neutron Radiotherapy Facility for Optimization of Neutron Capture Enhanced Fast-Neutron Therapy," Medical Physics 27:359$367,2000$.

12. R.E. Apfel, Y-C Lo, "Practical neutron dosimetry with superheated drops," Health Physics 56:79-83, 1979.

13. R. Nath, C. Meigooni, C. King, S. Smolen, F. d'Errico, "Superheated Drop Detector for Determination of Neutron Dose Equivalent to Patients Undergoing High-Energy X-Ray and Electron Radiotherapy," Medical Physics 20:78, 1993.

14. E.L Alpen, K.A. Frankel "Biological effectiveness of recoil protons from neutrons of energy $5 \mathrm{keV}$ to $5 \mathrm{MeV}$,". In: Soloway A.H., Barth R.F., Carpenter D.E. (eds.) Advances in Neutron Capture Therapy, Plenum Press, New York, pp 201-206, 1993. 
15. R.W. Roussin, "BUGLE-80 Coupled 47-Neutron, 20 Gamma-Ray, P3 Cross Section Library," DLC-75, Radiation Shielding Information Center, 1980.

16. W.N. McElroy, et al., "SAND-II Neutron Flux Spectra Determination by Multiple Foil Activation Iterative Method," AWRL-TR-67-41, Vol. 1-4, 1967.

17. E.L. Draper Jr., "Integral Reaction Rate Determinations - Part I: Tailored Reactor Spectrum Preparation and Measurement," Nuclear Science and Engineering, 48:22-30,1971.

18. F. d'Errico, V. Giusti, E. Nava, M. Reginatto, G. Curziom J. Capala, "Fast Neutron Spectrometry of BNCT Beams," In: W. Sauerwein, R. Moss, A. Wittig (eds.), Research and Development in Neutron Capture Therapy, Monduzi Editore, Bologna, Italy, pp 1139-1144, 2002.

19. O.K. Harling, et al., "The Fission Converter-Based Epithermal Neutron Irradiation Facility at the Massachusetts Institute of Technology, Nuclear Science and Engineering, 140, 223-240, 2002. 


\title{
TRANSPORT BENCHMARKS FOR PHYSICAL DOSIMETRY TO SUPPORT DEVELOPMENT OF FAST-NEUTRON THERAPY WITH NEUTRON CAPTURE AUGMENTATION
}

\author{
D.W. Nigg ${ }^{1}$, J.K. Hartwell ${ }^{1}$, C.A. Wemple ${ }^{1}$, and R. Risler ${ }^{2}$ \\ 1. Idaho National Engineering and Environmental Laboratory, Idaho Falls, ID \\ 2. University of Washington Medical Center, Seattle, WA
}

\section{INTRODUCTION}

The Idaho National Engineering and Environmental Laboratory (INEEL) and the University of Washington (UW) Medical Center are collaborating on efforts directed toward the development of supporting technologies required for advanced studies of fast-neutron therapy (FNT) with concurrent neutron capture (NCT) augmentation. ${ }^{1}$ In addition to the development of optimized neutron sources ${ }^{2}$, UW and INEEL researchers are conducting measurements to produce suitable benchmark neutron dosimetry data for validation of advanced three-dimensional treatment planning methodologies required for successful administration of FNT/NCT.

This experimental effort has included several related components. Free-beam spectral measurements using resonance and threshold activation foil techniques and measurements of dose-depth profiles and thermal neutron activation rates in a large water phantom have been completed. Extensive resonance and threshold activation foil measurements are also underway, using two cylindrical Lucite phantoms designed for increased relative sensitivity to the lowenergy tail of the neutron spectrum produced by the proton-cyclotron based UW neutron radiotherapy facility. Additional measurements are planned for the neutron therapy facility at the Fermi National Accelerator Laboratory, in Illinois, and at the University of Essen neutron therapy clinic in Germany. The results will be useful for validation of various explicit neutron transport codes and methodologies for FNT/NCT computational dosimetry, such as $\mathrm{MCNP}^{3}$ (Monte Carlo N-Particle), SERA ${ }^{4}$ (Simulation Environment for Radiotherapy Applications), and MINERVA $^{5}$ (Modality-Inclusive Environment for Radiotheraputic Variable Analysis). Some initial phantom measurement data for the UW facility are discussed in this report.

\section{METHODS AND MATERIALS}

Figure 1 shows the UW treatment gantry, with the larger of the two Lucite phantoms in position on the patient couch. This phantom has a diameter of $15.2 \mathrm{~cm}$ and a height of $21.6 \mathrm{~cm}$. The smaller phantom has corresponding dimensions of $12.7 \mathrm{~cm}$ and $18.2 \mathrm{~cm}$, respectively. The isocenter of the beam was located at the center of the top phantom surface. The field size at the isocenter was set to $7.8 \mathrm{~cm} \times 8.0 \mathrm{~cm}$ for all irradiations. The measurements presented here are for the case with the UW "standard" beryllium neutron production target mounted in the gantry. The proton beam current incident on the target was 67 microamperes, with a nominal proton energy of $50.5 \mathrm{MeV}$. Several different types of foil packages containing gold, manganese, aluminum, copper, and indium foils, with and without cadmium covers, were placed at various depths along the central axis of each phantom. The irradiation time in each case was generally 
one hour. After the completion of each irradiation, the induced activities resulting from the various interactions of interest in the foils were measured using standard gamma-ray spectrometry, and the results were converted to saturation activities, or, equivalently, activation rates per atom per microampere of proton current impinging on the neutron production target during the irradiation.
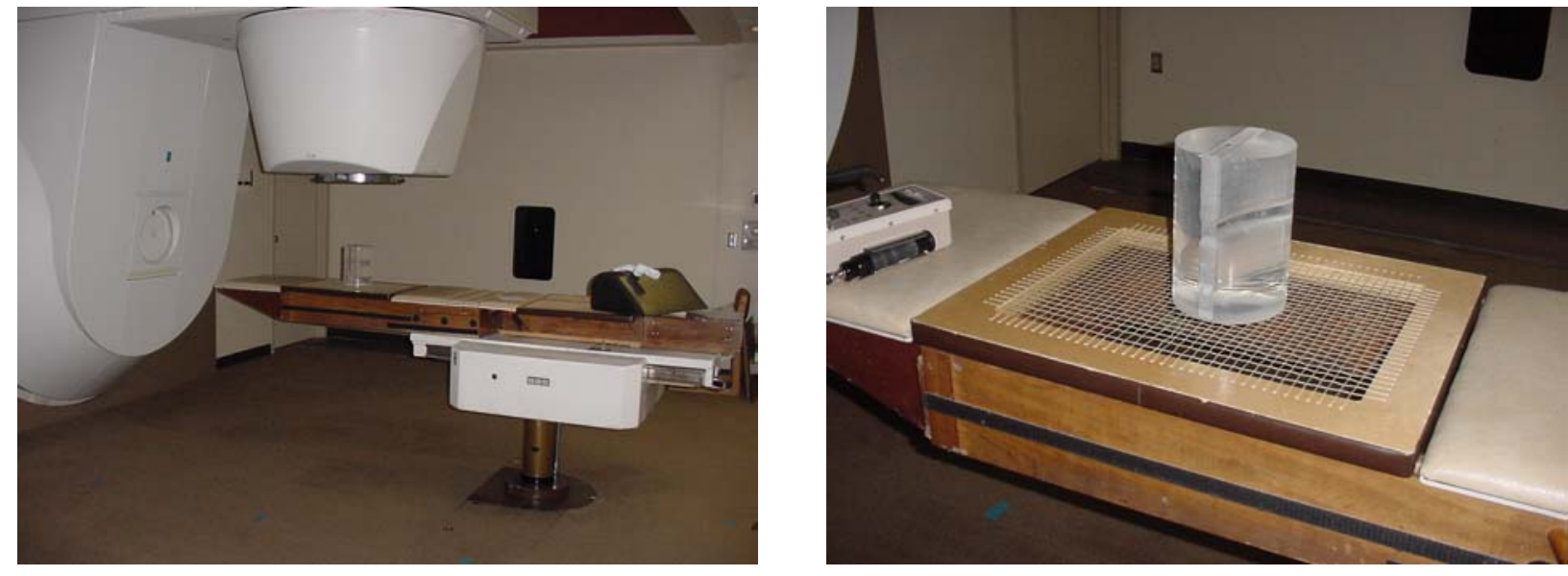

Figure 1. University of Washington neutron therapy treatment facility with the large cylindrical Lucite phantom in position.

\section{RESULTS AND DISCUSSION}

Figures 2 and 3 show some typical results. In Figure 2 the measured activation of manganese with and without cadmium covers is shown as a function of depth in each phantom. In the case of the bare foils, most of the activation is caused by thermal neutron absorption, and it can be seen that the thermal flux-depth curve is flatter for the larger phantom. This is expected, since this phantom is more effective at thermalizing the higher-energy neutrons in the beam. However, with cadmium covers on the dosimeter packages, the activation is largely caused by resonance neutron absorption at the primary resonance energy $(340 \mathrm{eV})$ and above in both phantoms, and the curves are basically the same.

Figure 3 shows the measured activity of various isotopes of gold as functions of depth. Production of ${ }^{192} \mathrm{Au}$ through ${ }^{196} \mathrm{Au}$ is via the $(\mathrm{n}, 6 \mathrm{n})$ through $(\mathrm{n}, 2 \mathrm{n})$ interactions, respectively. These interactions have thresholds spanning the energy range from $38.0 \mathrm{Mev}$ down to $8.6 \mathrm{MeV}$. Production of ${ }^{198} \mathrm{Au}$ from resonance capture in gold, primarily at $5 \mathrm{eV}$, is also shown. 

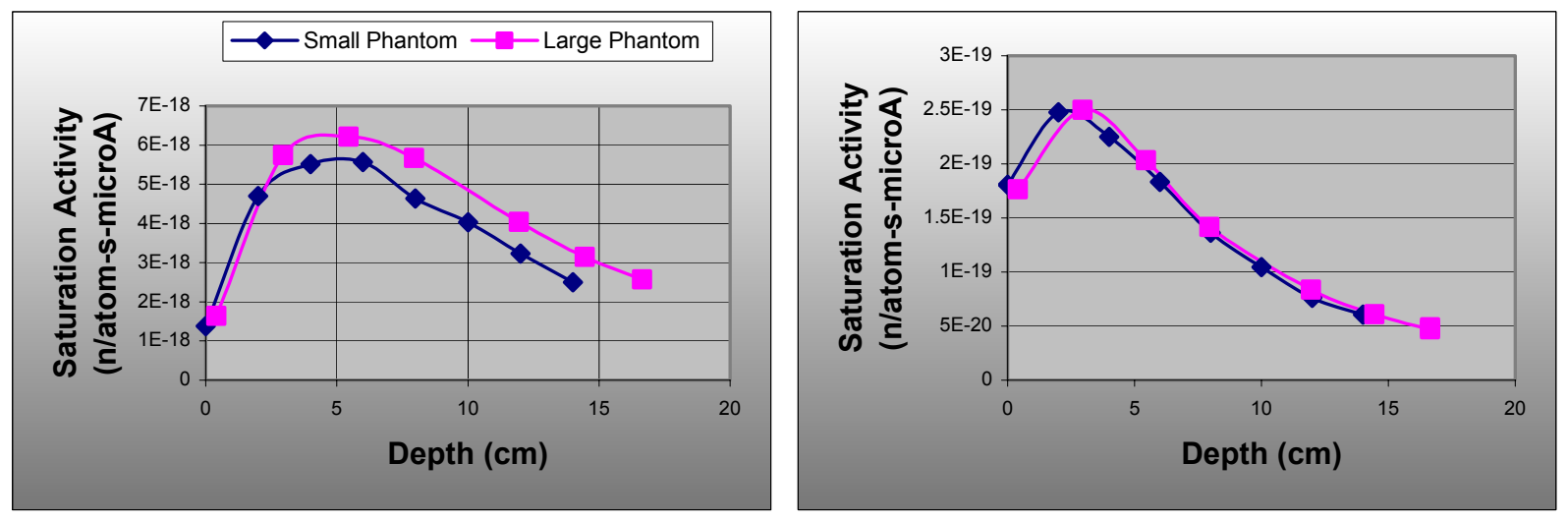

Figure 2. Saturation activities for Mn-56 in the small and large phantoms, without (left) and with (right) cadmium foil covers. Uncertainties are comparable to the size of the data points shown.

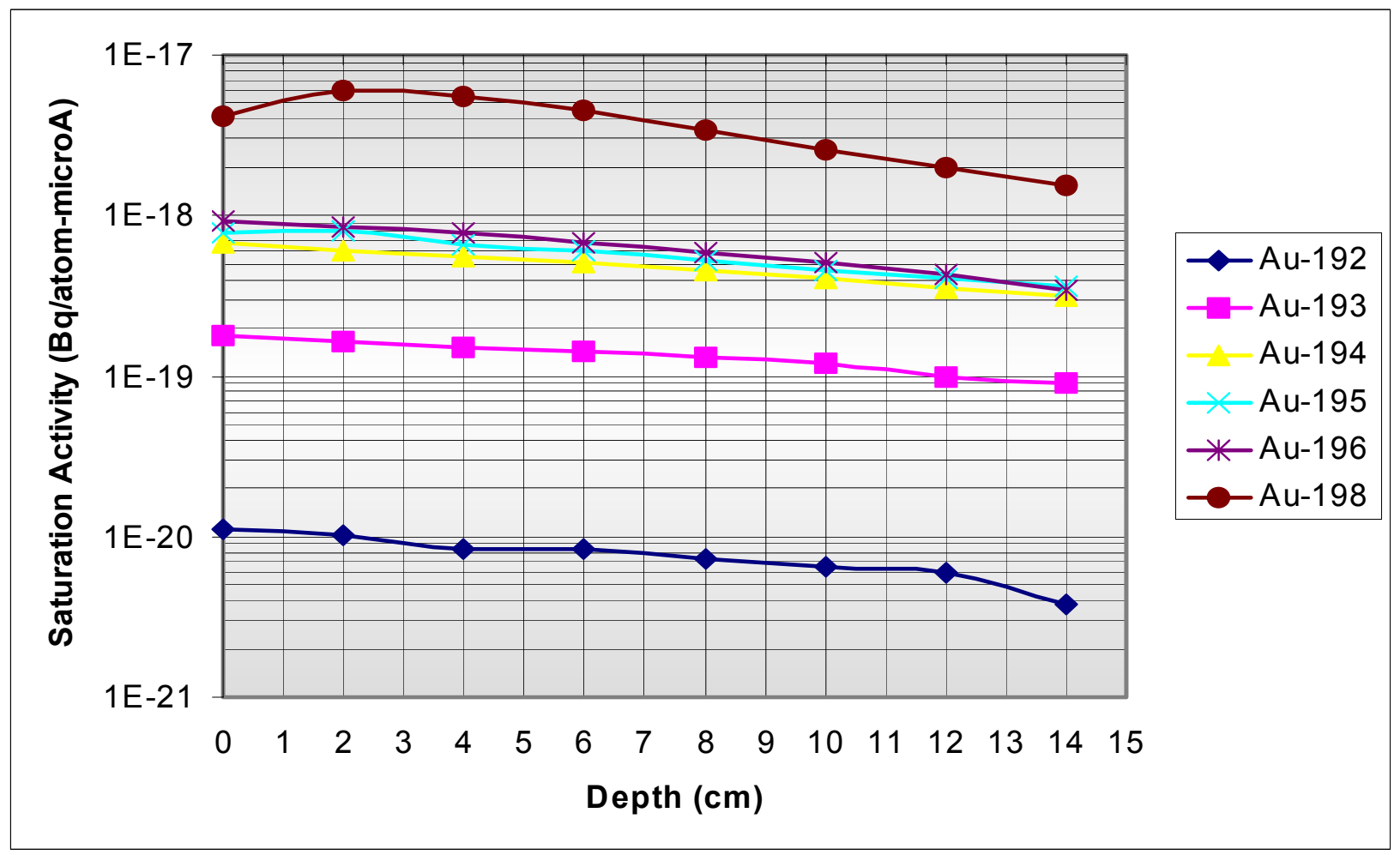

Figure 3. Saturation activities for several gold isotopes in the small phantom.

This information, along with additional information from the numerous other resonance and threshold interactions in the other foils, allows the development of a self-consistent picture of the space- and energy-dependent behavior of the neutron flux within the two phantoms. It is also of interest in this work to develop methods of reconstructing the incident beam spectrum based on the measured activation rates in conjunction with a computed response matrix for each phantom. 


\section{REFERENCES}

1. G.E. Laramore, P. Wootton, J.C. Livesey, D.S. Wilbur, R. Risler, M. Phillips, J. Jacky, T.A. Bucholtz, T.W. Griffin, S. Brossard, "Boron neutron capture therapy - A mechanism for achieving a concomitant tumor boost in fast neutron radiotherapy," Int. J. Rad. Onc. Bio. Phys., 28:1135-1142, 1994.

2. D.W. Nigg, C.A. Wemple, R. Risler, J.K. Hartwell, Y.D. Harker, G.E. Laramore, "Modification of the University of Washington Neutron Radiotherapy Facility for optimization of neutron capture enhanced fast-neutron therapy," Medical Physics, 27:359$367,2000$.

3. J.F. Briesmeister, "MCNP - A General Monte Carlo N-Particle Transport Code, Version 4A," LA-12625-M, Los Alamos National Laboratory, 1993.

4. D.W. Nigg, C.A. Wemple, D.E. Wessol, F.J. Wheeler, "SERA-An Advanced Treatment Planning System for Neutron Therapy and BNCT," Trans. ANS, 80:66-68, 1999.

5. D.W. Nigg, "Computational Dosimetry and Treatment Planning Considerations for Neutron Capture Therapy," In Publication, J. Neuro Oncology, 2003. 


\title{
COLLABORATIVE IN-PHANTOM PERFORMANCE CHARACTERIZATION OF THE CNEA HYPERTHERMAL NEUTRON BEAM FACILITY FOR BNCT
}

\author{
D.W. Nigg ${ }^{1}$, H Blaumann ${ }^{2}$, O.C. Larrieu ${ }^{2}$, J. Longhino ${ }^{2}$, C.A. Wemple ${ }^{1}$, S. Ribeiro ${ }^{2}$, and M. \\ Arribere $^{2}$
}

1. Idaho National Engineering and Environmental Laboratory, Idaho Falls, ID

2. Comision Nacional de Energia Atomica, Centro Atomico Bariloche, San Carlos de Bariloche, Argentina

\section{INTRODUCTION}

The National Atomic Energy Commission of Argentina (CNEA) has constructed a neutron beam facility for Boron Neutron Capture Therapy (BNCT) applications at their laboratory complex near San Carlos de Bariloche. The RA-6 research reactor at this installation is used in conjunction with a neutron filtering, moderating, and beam extraction structure located adjacent to the reactor core to produce an epithermal neutron source and, with some minor modification, a hyperthermal neutron source suitable for preclinical research as well as selected clinical applications. The primary neutron filtering and moderating materials used in the beam line are aluminum and aluminum oxide. Figure 1 shows the basic beam extraction component design. The acrylic section just downstream of the final bismuth gamma shield can be inserted or removed to produce a hyperthermal or epithermal neutron beam, as desired. Figure 2 shows the beam port and treatment room interior.

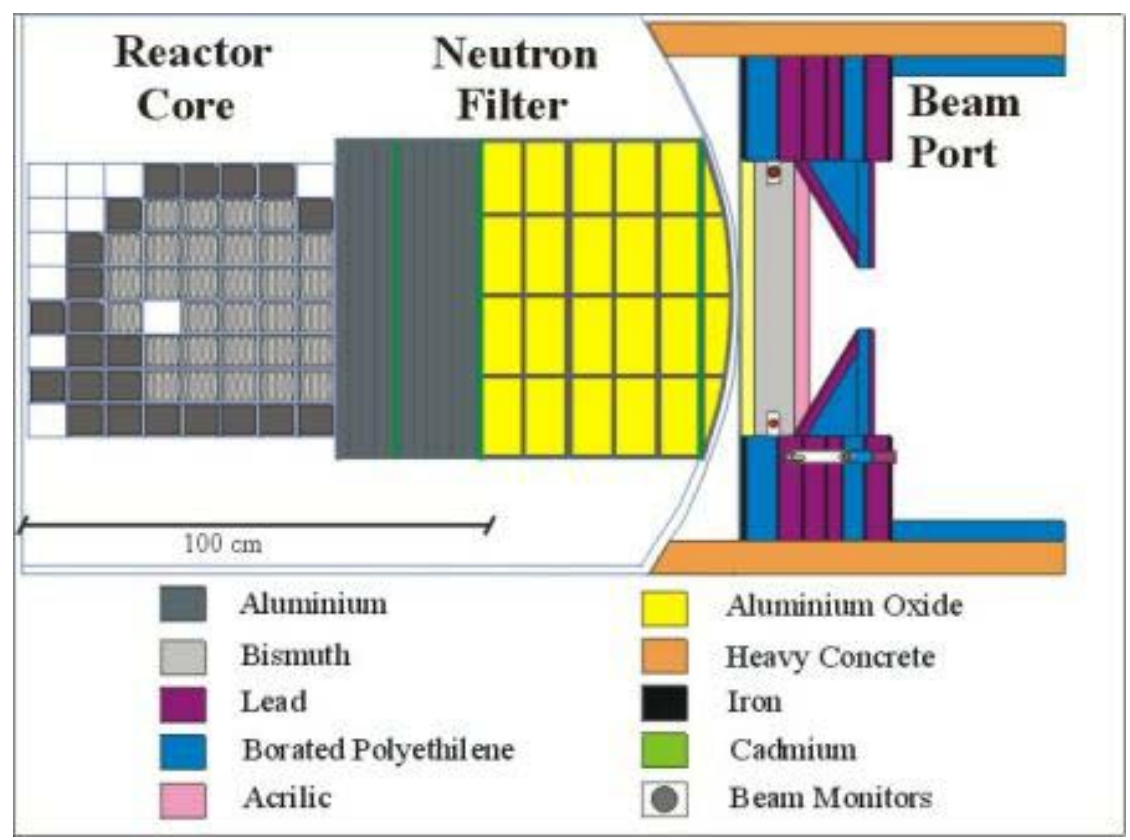

Figure 1. RA-6 neutron beam filtering and extraction component design. 
The Idaho National Engineering and Environmental Laboratory (INEEL), in collaboration with CNEA under a bilateral U.S./Argentina agreement on peaceful uses of nuclear science, has conducted some basic neutron beam characterization measurements at the new RA-6 facility. Results for the epithermal configuration were summarized in a previous Annual Report. ${ }^{1}$ The results for the hyperthermal configuration are summarized in this article.

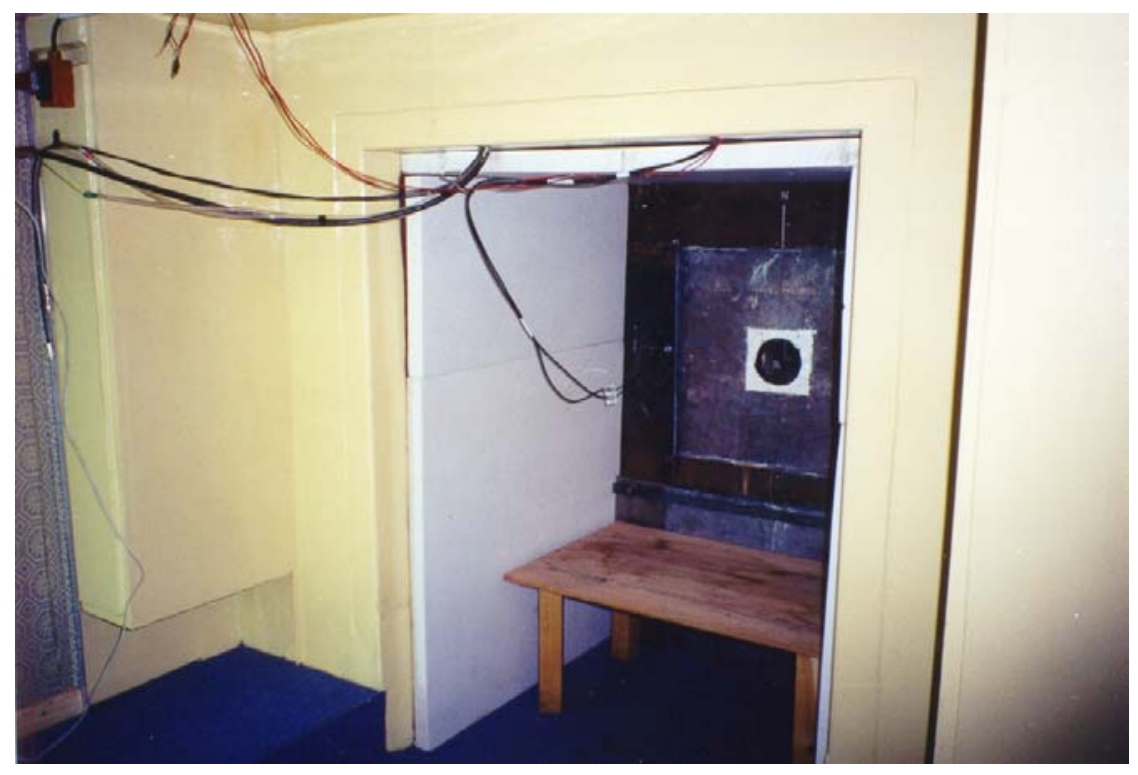

Figure 2. RA-6 epithermal neutron beam port.

\section{METHODS AND MATERIALS}

The various measurements were performed using simplified extensions of neutron activation analysis techniques ${ }^{2,3}$ that have been adapted for BNCT applications by the INEEL through extensive experience with several other medical neutron source facilities in the U.S. and in Europe. Auxiliary neutron transport computations of various types are also required as part of the experimental protocol. CNEA performed some of these computations, while others were performed by the INEEL, using the well-known $\mathrm{MCNP}^{4}$ code.

Measurements of the neutron flux-depth distribution produced by the RA-6 hyperthermal beam in a standard water phantom were performed using activation techniques. Figure 3 shows the phantom, a Lucite box of dimension $400 \times 400 \mathrm{~mm}$ in height and width, and $200 \mathrm{~mm}$ depth along the beam axis. The wall thickness of the phantom on the beam entrance side is $5 \mathrm{~mm}$. The phantom was positioned against the RA-6 beam exit port, as shown. The flux wires used for the measurements reported here were approximately $10 \mathrm{~mm}$ in length and $1 \mathrm{~mm}$ in diameter. Each wire had a mass of approximately $90 \mathrm{mg}$ and was composed of copper alloyed with 1.55 percent gold by weight. This provided two linearly-independent activation responses (neutron absorption in ${ }^{63} \mathrm{Cu}$ and ${ }^{197} \mathrm{Au}$ ) that were used to unfold a two-group (thermal and above-thermal) neutron spectrum at each flux wire location. Flux wires were placed at axial depths of 0, 6, 20, 
$30,40,50,60$, and $70 \mathrm{~mm}$ along the central axis, and at radii of 10,30, 50, 70, 90, 110, and 130 $\mathrm{mm}$ at a depth of $6 \mathrm{~mm}$.

The measured activation rates of the foils and wires were used to estimate the neutron spectrum of the RA- 6 beam and the fluxes in the water phantom by way of a direct unfolding procedure adapted by the INEEL for this type of measurement. ${ }^{5}$ The required unfolding parameters for the flux wire materials were computed at each axial location in the phantom using an MCNP model of the phantom, with the RA-6 neutron source (also computed by MCNP) incident on the side.

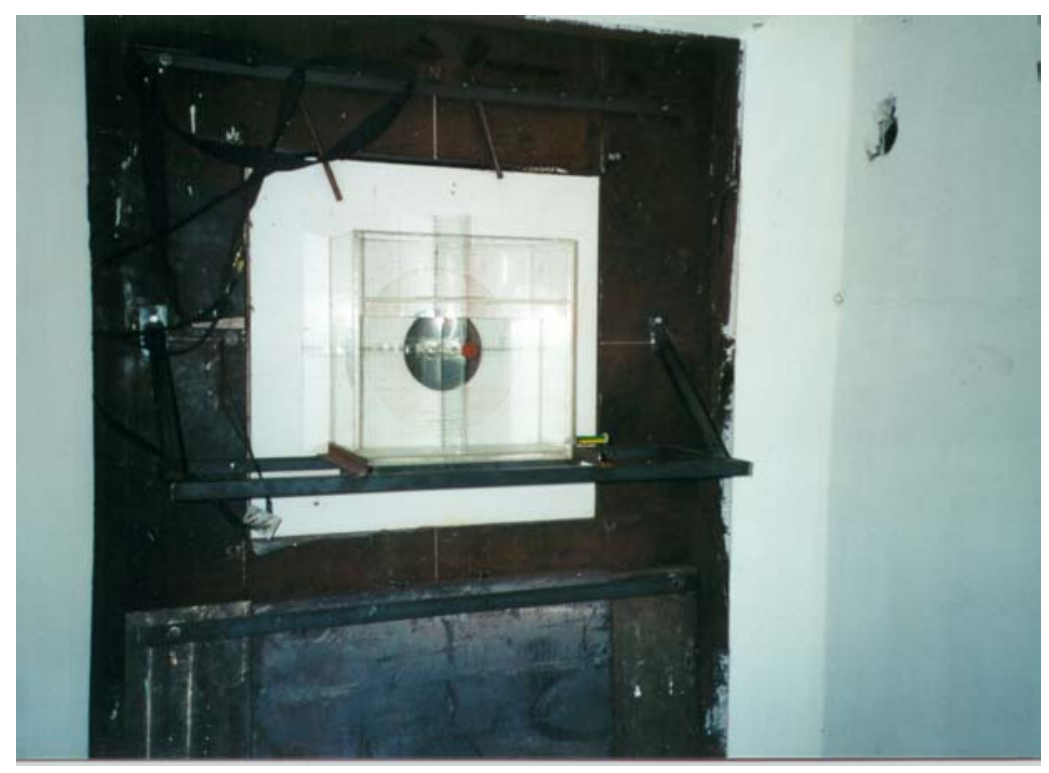

Figure 3. Water phantom positioned in the RA-6 beam port.

\section{RESULTS}

Figures 4 and 5 show the unfolded thermal $(\mathrm{E}<0.414 \mathrm{eV})$ and above-thermal neutron fluxes at the various depths of interest along the central phantom axis and as a function of radius at a depth of $6 \mathrm{~mm}$. An independent set of measurements for the thermal flux in both cases, completed earlier by CNEA using the cadmium difference method, is also shown for comparison. The CNEA measurements were based on the use of thin gold wires, with casespecific MCNP computations to determine the parameters required to estimate the thermal neutron flux from the wire activities. Agreement is quite good, especially considering the independence of the two sets of measurements.

\section{DISCUSSION}

The RA-6 facility is the first clinical scale neutron source for neutron capture therapy research in Latin America and one of only about 10 such facilities currently operational in the world. The results presented here independently confirm the neutronic performance of the RA-6 neutron beam using a very simple experimental approach. 


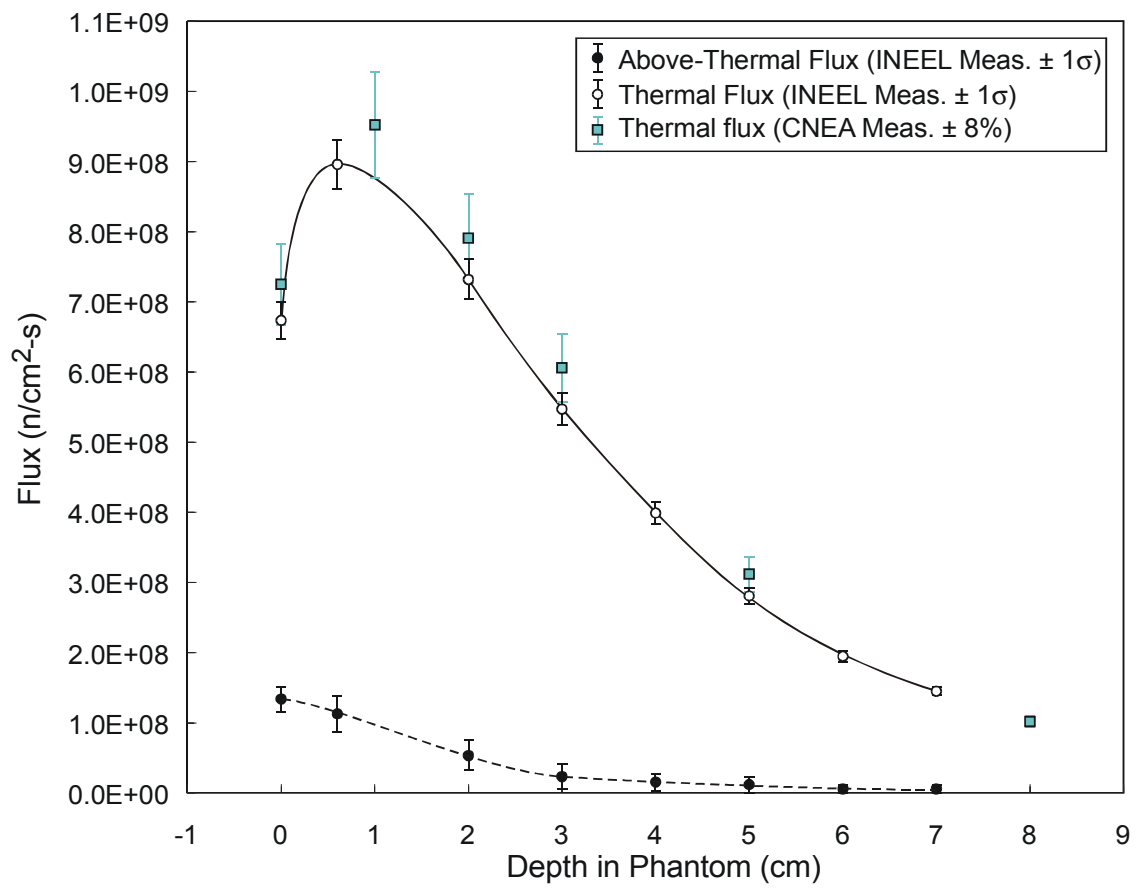

Figure 4. Measured thermal and above-thermal neutron fluxes along the central axis of the CNEA water phantom. Uncertainties of the measured points are shown at the $1 \sigma$ level. The reactor power was $360 \mathrm{~kW}$.

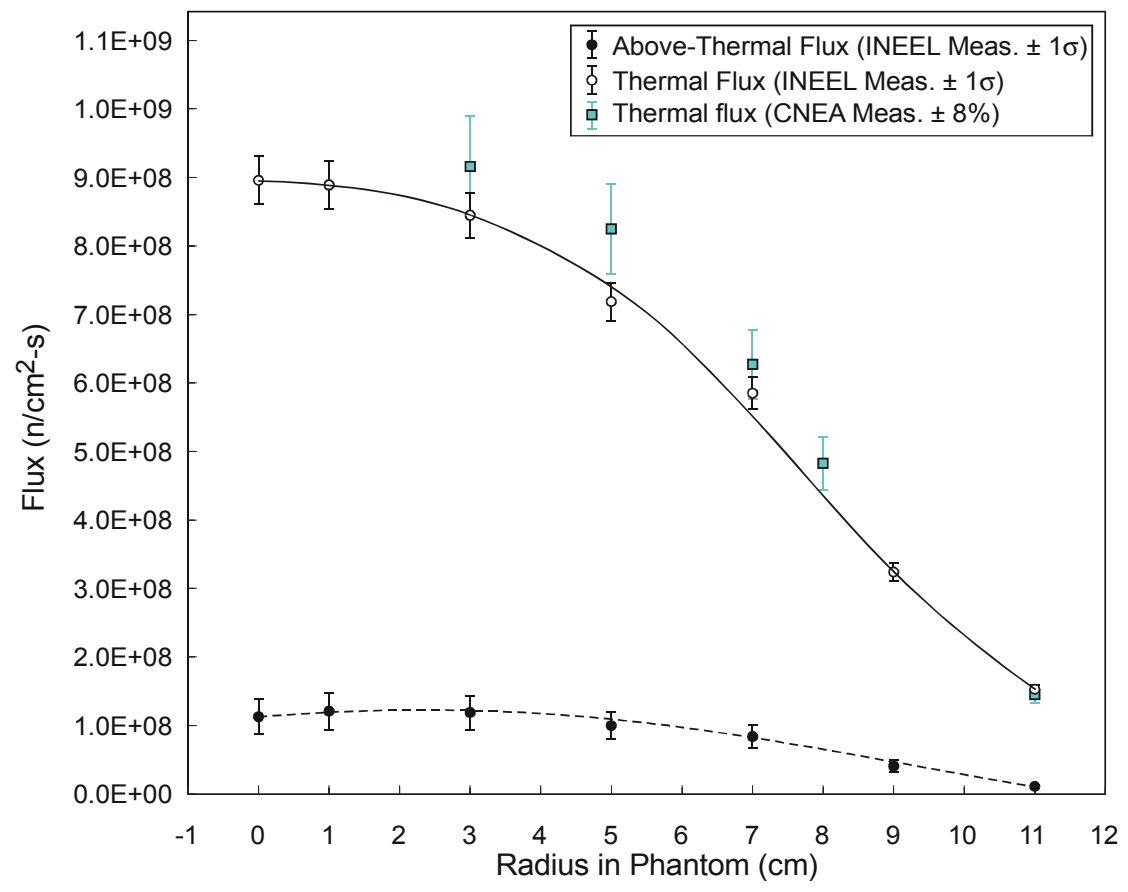

Figure 5. Measured thermal and above-thermal neutron fluxes along the radial dimension at an axial depth of $6 \mathrm{~mm}$ in the CNEA water phantom. Uncertainties of the measured points are shown at the $1 \sigma$ level. The reactor power was $360 \mathrm{~kW}$. 


\section{ACKNOWLEDGEMENTS}

INEEL participation in this work was sponsored by the U.S. Department of Energy, Office of Nuclear Nonproliferation (NN-44), through the Sister Laboratory Program at Argonne National Laboratory (ANL). The INEEL and CNEA participants would like to acknowledge the assistance and support of Dr. Basil Picologlou (ANL) and Dr. Sara Liberman (CNEA) in particular.

\section{REFERENCES}

1. D.W. Nigg, H. Blaumann, O. Calzetta Larrieu, J. Longhino, C.A. Wemple, S. Ribeiro, "Collaborative Neutronic Performance Characterization of the CNEA Epithermal-Neutron Beam Facility for BNCT," In: J.R. Venhuizen (ed.), INEEL BNCT Research Program Annual Report 2000, INEL/EXT-01-00204, March 2001, pp 70-83.

2. D.W. Nigg, et al., "Collaborative Neutronic Performance Characterization of the FiR 1 Clinical Epithermal-Neutron Beam Facility for BNCT," In: J.R. Venhuizen (ed.), INEEL BNCT Research Program Annual Report 1998, INEL/EXT-99-00293, April 1999, pp 13-38.

3. Y.D. Harker, et al., "Spectral Characterization of the Epithermal Neutron Beam at the Brookhaven Medical Research Reactor," Nuclear Science and Engineering, 110:355-368, 1992.

4. J.F. Briesmeister, "MCNP - A General Monte Carlo N-Particle Transport Code, Version 4A,” LA-12625-M, Los Alamos National Laboratory, 1993.

5. D.W. Nigg, C.A. Wemple, R. Risler, J.K. Hartwell, Y.D. Harker, G.E. Laramore, "Modification of the University of Washington Neutron Radiotherapy Facility for Optimization of Neutron Capture Enhanced Fast-Neutron Therapy," Medical Physics, 27:359-367, 2000. 